\title{
A review of Cameroonian medicinal plants with potentials for the management of the COVID-19 pandemic
}

\author{
Evariste Fongnzossie Fedoung 1,2,4,5,10 . Achille Bernard Biwole ${ }^{1}$. Christine Fernande Nyangono Biyegue ${ }^{2}$. \\ Marlene Ngansop Tounkam ${ }^{3,4}$. Patrick Akono Ntonga ${ }^{6}$. Véronique Priscille Nguiamba ${ }^{10}$. \\ Damien Marie Essono ${ }^{3}$. Preasious Forbi Funwi ${ }^{3,4}$. Calvin Tonga ${ }^{6}$. Guy Merlin Nguenang ${ }^{3,5}$. Victor Kemeuze ${ }^{4,5}$. \\ Denis Jean Sonwa ${ }^{7} \cdot$ Nole Tsabang $^{4,5} \cdot$ Isabelle Sandrine Bouelet ${ }^{2} \cdot$ Zra Tize $^{2}$. Alexandre Teplaira Boum ${ }^{2}$. \\ Marie Caroline Momo Solefack ${ }^{5,8}$. Jean Lagarde Betti ${ }^{9} \cdot$ Achille Nouga Bissoue $^{2}$. Leopold Gustave Lehman ${ }^{6}$. \\ Pierre Marie Mapongmetsem $\mathrm{m}^{4,5} \cdot$ Leandre Nneme Nneme $^{10} \cdot$ Rosalie Annie Ngono Ngane $^{11}$. \\ Jeanne Ngogang Yonkeu ${ }^{5,12,13}$
}

Received: 10 September 2020 / Accepted: 18 February 2021 / Published online: 26 March 2021

(c) Institute of Korean Medicine, Kyung Hee University 2021

\begin{abstract}
Since the outbreak in December 2019, in Wuhan (China) of COVID-19, approved drugs are still lacking and the world is seeking effective treatment. The purpose of this article is to review the medicinal plants with potential to be used as complementary therapies against COVID-19. Bibliographic information was searched in several databases (Google Scholar, PubMed, Scopus, ScienceDirect, PROTA, ResearchGate and GLOBEinMED), to retrieve relevant papers on (1) plants used to manage common symptoms of COVID-19, (2) plant secondary metabolites with confirmed inhibitory effects on COVID-19 and (3) plants exhibiting pharmacological activities of relevance for COVID-19 management. A total of 230 species was recorded as potential source of ingredients for the fight against the 2019 novel corona virus. Of these species, 30 contain confirmed antiCOVID-19 secondary metabolites, 90 are used traditionally to manage at least 3 common symptoms of COVID-19, 10 have immunostimulant activity, 52 have anti-inflamatory activity, 14 have antiviral properties and 78 species are documented as used to treat malaria. A PCA analysis showing cluster formatting among the recorded species indicates 4 groups of species and an array of possibility of using individual species or a combination of species for their complementary effects. The authors argue that Cameroonian medicinal plants can be of potential contribution to the fight against COVID-19. Further applied research is needed to provide more scientific evidence for their efficacy, to establish standard formulations and clinical studies as part of efforts to develop therapies for COVID-19.
\end{abstract}

Keywords Medicinal plants $\cdot$ Ethnobotany $\cdot$ Phytochestry $\cdot$ Pharmacological properties $\cdot$ Review

Evariste Fongnzossie Fedoung

fongnzossie@gmail.com

1 Laboratory of Forest Resources and Wood Valorization, University of Douala, PO Box 1872, Douala, Cameroon

2 Laboratory of Process Engineering, University of Douala, PO Box 1872, Douala, Cameroon

3 Laboratory of Botany, University of Yaoundé 1, PO Box 812, Yaoundé, Cameroon

4 Millenium Ecologic Museum, PO Box 8038, Yaoundé, Cameroon

5 Cameroon Ethnobotany Network, PO Box 8038, Yaoundé, Cameroon

6 Laboratory of Animal Biology, Department of Animal Biology, Faculty of Science, University of Douala, P.O. Box 2701, Douala, Cameroon
7 Center for International Forestry Research, PO Box 2008, Messa, Yaoundé, Cameroon

8 Laboratory of Applied Botany, University of Dschang, PO Box 67, Dschang, Cameroon

9 Laboratory of Plant Biology and Physiology, University of Douala, P.O. Box 24157, Douala, Cameroon

10 Advanced Technical Teachers Training School for Technical Education, University of Douala,, PO Box 1872, Douala, Cameroon

11 Laboratory of Biochemestry, University of Douala, PO Box 24157, Douala, Cameroon

12 Cameroon Academy of Science, Douala, Cameroon

13 Université des Montagnes, Bangangté, Cameroon 


\section{Introduction}

\section{Background on the outbreak and epidemiology of the COVID-19 pandemic}

Corona viruses are well known in veterinary medicine. First discovered in the 1960s as parasites of infectious chicken bronchitis, they were later found to be responsible for serious epidemics in humans such as Severe Acute Respiratory Syndrome (SARS) in 2002/2003 and the Middle East Respiratory Syndrome (MERS) in 2012.

Huang et al. (2020) reported in late December 2019, an outbreak of a mysterious pneumonia of unknown cause in the Huanan Seafood Wholesale Market, in Wuhan, Hubei, China. The causal agent of this disease was isolated and identified by Chinese scientists as a new strain of Corona virus, the SARS-CoV-2 or 2019 novel corona virus (2019nCov). Data obtained on patients with laboratory-confirmed 2019-nCoV infection in the hospital of Wuhan indicated that the common early symptoms of this disease were fever ( $98 \%$ of patients), cough (76\%), and myalgia or fatigue (44\%). Complications associated with this disease as observed in hospitalized patients included acute respiratory distress syndrome (29\%), RNAaemia (15\%), acute cardiac injury (12\%) and secondary infections (10\%).

Because this 2019-nCov is spread by human-to-human transmission via droplets or direct contact (Lai et al. 2020), its emergence in China has caused a large global outbreak. According to the European Centre for Disease Prevention and Control, the worldwide situation update shows that since 31 December 2019 and as of 13 January 2021, a total of 84532 824cases of COVID-19 have been reported worldwide, including 1,845,597 deaths (ECDC 2020). During the same period, the African continent has reported 2,832,753 cases (26,846 cases reported for Cameroon and 448 deaths); the countries reporting with the greatest number of reported deaths included South Africa (29,577), Egypt (7805), Morocco (7485), Tunisia (4800) and Algeria (2772).

Despite the ongoing efforts to manage the disease, no antiviral drug currently exists for the prevention or treatment (Shio-Shin et al. 2020), and many months may be required for their development. There are actually a number of COVID-19 candidate vaccines for which certain national regulatory authorities have authorized their evaluation or use. However, none have yet received WHO authorization (Calina et al. 2020). However, the spread of the COVID-19 pandemic is very dynamic and growing around the world. In response to this outbreak, the World Health Organization, on January 30, 2020 declared that the pandemic constitutes a public health emergency of international concern and issued temporary recommendations under the International Health Regulations.

\section{Global therapeutic response to COVID-19}

Currently, no approved drug for COVID-19 exists and treatments provided worldwide to the affected persons are symptom based. These include antiviral drugs so far used against major groups of viruses like human immunodeficiency virus (HIV), herpes, hepatitis, influenza, SARS-CoV and MERS-CoV, antimalaria drugs, imunostimulants, antiinflammatory drugs that may be effective against elevated levels of cytokines and useful in inhibiting viral infection (Vellingiri et al. 2020).

Reviews by Vellingiri et al. (2020), Liu et al. (2020) and Wu et al. (2020) reported that the current most clinically used drugs can be grouped into three categories: antiviral agents, supporting agents and miscellaneous agents and therapies (Table 1).

Worldwide, a number of drugs which have so far been proven to be safe for humans, are currently being repurposed to be used for the management of this disease.

The 2019 novel coronavirus genome encodes several structural proteins, including the glycosylated spike (S) protein that functions as a major inducer of host immune responses. This $\mathrm{S}$ protein mediates host cell invasion via binding to a receptor protein called angiotensin-converting enzyme 2 (ACE2) located on the surface membrane of host cells. Hence, the interaction between viral $\mathrm{S}$ protein and ACE2 on the host cell surface is of significant interest in the therapeutic response process since it initiates the infection process.

\section{Herbal medicine and the COVID-19 challenge: a global overview}

Globally, herbal treatments have been proven effective to control contagious disease during the 2003 severe acute respiratory syndrome (SARS) outbreak (Zhang et al. 2020). Therefore, since the outbreak of COVID-19, there has been great attention in investigating metabolites secreted by plants that may be developed as medicines for COVID-19.

Historically, traditional medicine and local beliefs have always played a role in epidemics through time (Zhang 1996). A review by Jassim and Naji (2003) reported numerous potentially useful medicinal plants that need to be evaluated and exploited for therapeutic applications against genetically and functionally diverse virus families. Keyaerts et al. (2007) identified a variety of plant lectins as antiviral compounds against the SARS-CoV. Lelesius et al. (2019) also showed that some extracts of plants including Thymus vulgaris and Desmodium canadense were effective against avian infectious bronchitis virus, a highly contagious respiratory disease in chickens caused by a corona virus that belongs to the Coronaviridae family. From 
Table 1 Current Therapeutic Drugs used in the treatments of COVID-19

\begin{tabular}{|c|c|}
\hline Drug name/category & Description \\
\hline \multicolumn{2}{|l|}{ Antiviral agents } \\
\hline Remdesivir & $\begin{array}{l}\text { A broad-spectrum antiviral agent, anti-viral peptide that was used in treatments against Ebola, SARS- } \\
\text { CoV and MERS-CoV }\end{array}$ \\
\hline Hydroxychloroquine and Chloroquine & $\begin{array}{l}\text { An anti-malarial drug, which has been effective in the treatment of avian influenza A; } \\
\text { Can inhibit the entry of SARSCoV-2 and prevent virus-cell fusion by interfering with glycosylation of } \\
\text { the ACE2 receptor and its binding with spike protein; } \\
\text { Anti-viral and immune modulating properties; } \\
\text { Can reduce cytokine storm }\end{array}$ \\
\hline Lopinavir-Ritonavir & $\begin{array}{l}\text { Lopinavir is a protease inhibitor with high specificity for HIV1 protease } \\
\text { Ritonavir inhibits the enzymes that are responsible for lopinavir metabolism, and its co-administration } \\
\text { improves antiviral activity } \\
\text { Lopinavir was also used along with another flu drug, oseltamivir and resulted in complete recovery of } \\
\text { patients showing signs of COVID-19 related pneumonia }\end{array}$ \\
\hline Umifenovir (Arbidol) & $\begin{array}{l}\text { A drug targeting } \mathrm{S} \text { protein/ACE2, it is an inhibitor that may disrupt the binding of viral envelope protein } \\
\text { to host cells, thus preventing viral entry to the target cell. It has been used earlier as influenza antiviral } \\
\text { drug; } \\
\text { An in vitro antiviral efficacy in widely spreading virus strains such as the Ebola virus, human herpesvi- } \\
\text { rus } 8 \text { (HHV-8), hepatitis C virus (HCV), and Tacaribe arenavirus }\end{array}$ \\
\hline Favipiravir (Avigan) & $\begin{array}{l}\text { A broad spectrum anti-viral drug, a purine nucleoside whose possible mechanism on COVID-19 is } \\
\text { through its action as an alternate substrate leading to inaccurate viral RNA synthesis }\end{array}$ \\
\hline Oseltamivir (Tamiflu) & $\begin{array}{l}\text { A drug approved for treatment of influenza A and B. It inhibits the spread of the influenza virus in the } \\
\text { human body }\end{array}$ \\
\hline Ribavirin & $\begin{array}{l}\text { A broad-spectrum antiviral drug used in the treatment of hepatitis } \mathrm{C} \text {, in combination with interferon } \alpha \\
\text { (IFN) }\end{array}$ \\
\hline Sofosbuvir & A drug also used for the treatment of hepatitis $\mathrm{C}$ in combination with interferon or RBV \\
\hline \multicolumn{2}{|l|}{ Supporting agents } \\
\hline Azithromycin & $\begin{array}{l}\text { An antibiotic used against many different types of infections caused by susceptible bacteria, } \\
\text { It has been proven to be active in vitro against Zika and Ebola viruses and to prevent severe respiratory } \\
\text { tract infections when administered to patients suffering viral infection }\end{array}$ \\
\hline Vitamin C (Ascorbic Acid) & $\begin{array}{l}\text { As a potent antioxidant agent, it neutralizes free radicals and helps to prevent or reverse cellular damage } \\
\text { It has immunomodulatory activity } \\
\text { An antiviral agent against influenza viruses }\end{array}$ \\
\hline Zinc & $\begin{array}{l}\text { Inhibits SARS-CoVand retrovirus RNA polymerase activity } \\
\text { Enhances chloroquine intracellular uptake }\end{array}$ \\
\hline Corticosteroids & $\begin{array}{l}\text { Anti-inflammatory drug } \\
\text { Prevent an extended cytokine response and may accelerate resolution of pulmonary and systemic inflam- } \\
\text { mation in pneumonia }\end{array}$ \\
\hline Tocilizumab (Actemra) & $\begin{array}{l}\text { Immunosuppressive drug, emerged as an alternative treatment for COVID-19 patients with a risk of } \\
\text { cytokine storms }\end{array}$ \\
\hline Sarilumab & $\begin{array}{l}\text { It inhibits IL- } 6 \text { that might play a role in driving the inflammatory immune response that causes acute } \\
\text { respiratory distress syndrome observed in patients with severe COVID-19 infection }\end{array}$ \\
\hline Interferon (IFN) Beta 1-Alpha) & It has nonspecific antiviral as well as other complex effects on immunity and cell proliferation \\
\hline Miscellaneous Agents and Therapies & \\
\hline Ibuprofen & Activators of ACE2 receptors \\
\hline Indomethacin & Antiviral activity against SARS-CoV and canine coronavirus (CCoV) \\
\hline
\end{tabular}

Adapted from Vellingiri et al. (2020), Liu et al. (2020) and Wu et al. (2020)

all over the world, people are witnessing a deep attachment to popular medicine to protect themselves against COVID-19. This is because to date, herbal products have proven to be not only effective, but also widely available to consumers.
Africa is endowed with diverse environmental conditions and a diversity of pathogenic microbial species (bacteria, fungi, and viruses). These microbes are causal agents of a great number of diseases (Cunningham et al. 2008), thus suggesting that African plants could accumulate 
chemopreventive substances more than plants from the northern hemisphere (Mahomoodally 2013). Basically, more than $80 \%$ of the population in this continent is known to rely on traditional medicine for their primary health care needs.

In Burkina Faso, the country's plan to respond to the COVID-19 pandemic does not rule out the use of herbal medicines, and clinical trials are underway on Apivirine, a phytomedicine from Benin which is alleged to be effective against the coronavirus (Sputniknews 2020).

In Algeria, to face the spread of this pandemic, consultation of herbalists in the search of traditional antiviral and anti-flu recipes have significantly increased (Le Point International 2020).

Goothy et al. (2020) supported the possible role of medicinal plants in Ayurveda's medicine for the management of Corona virus disease (COVID-19). Sharma and Kaur (2020) showed that Jensenone from Eucalyptus essential oil was a potential inhibitor of 2019 corona virus.

In China, DU Hong-Zhi et al. (2020) argued that traditional chinese medicine is an effective treatment for the 2019 novel coronavirus pneumonia.

More recently, the Malagasy Institute for Applied Research developed an herbal tea based on Artemisia аппиа (COVID Organics), claiming preventive and curative properties against COVID-19 (Midi-Madagascar 2020).

In China, herbal traditional medicine have been proven effective to control contagious disease during the 2003 severe acute respiratory syndrome (SARS) outbreak and a recent screening of a Chinese herbal medicine database have confirmed that herbal treatments classically used for treating viral respiratory infection contain chemical compounds that have potential anti-2019-nCoV activity (Zhang et al. 2020).

In Nigeria, recent reviews on potential plants for treatment and management of COVID-19 have been carried out. The results presented up to a hundred Nigerian indigenous medicinal plants with therapeutic abilities which may serve as effective treatments for COVID-19 due to their antiviral, anti-inflammatory, antioxidant, antipyretic, immunomodulatory and cyto-protective properties (Oladele et al. 2020; Ikpa et al. 2020).

In Cameroon, since the first case was reported in the country, several herbal recipes have been popularized in social media, as alleged solutions to manage COVID 19. According to a recent release from the Cameroon Radio and Television Corporation, the Archbishop of Douala, His grace Samuel Kleda, has made public an attempt at treating symptoms of COVID-19 with a herbal remedy, free of charge and the Ministry of Public Health is showing commitment to support the process of development and homologation of this treatment (Crtv 2020).

As the world is currently seeking treatment for COVID19 , there is an urgent need to boost up research so as to develop effective and affordable therapeutics.

\section{Cameroon's response strategy to COVID-19}

In Cameroon, access to health care services is challenging. One out of every 1,000 patients is able to see a specialist and 3 out of 20 patients are able to buy prescribed drugs in hospitals (Kuete and Efferth 2010). In this context, the COVID-19 situation is likely to worsen as the country moves into phase 2 of this pandemic marked by a shift from virus importation to intra-community transmission. Based on this situation, the Government prepared a COVID-19 Preparedness and Response Plan of US\$600 million to respond to the crisis, under the leadership of the Ministry of Public Health and with the partnership of international organizations. This health response strategy has eight components:

- Multisectoral and international coordination,

- Surveillance for early detection of cases,

- Investigation and rapid intervention teams,

- Laboratory capacities,

- Infection prevention and control measures in hospitals and in the community,

- Case management,

- Risk communication and community engagement, and

- Logistics.

Several treatment protocols including the Chloroquinebased treatment suggested by Professor Didier Raoult (Colson et al. 2020) are being tested with varying degree of effectiveness.

However, since the outbreak of this disease, ethnobotanical and ethnopharmacological research geared at bringing the potentials of traditional medical knowledge into the debate over the management of this disease has been lacking. Yet Cameroon is a biologically diverse country. This country is located in Central Africa, in the heart of the Congo Basin, the world's second largest rainforest after the Amazon. Its floristic potential scores more than 7850 plant species recorded at the national herbarium. This ranks Cameroon among the countries with the highest levels of biodiversity in Africa. Despite the inaccuracy of statistics, medicinal plants are important elements of health care services. However, access to such plants has so far been largely through traditional healers and herbal markets which are part of an informal economy. The huge volume of published research on medicinal plants in Cameroon surprisingly contrasts with the paucity of approved phytodrugs. Among the pressing challenges that must be tackled for acceptable use of traditional and alternative medicines in modern therapeutics in Cameroon are:

- the increasing use of traditional medicines and the general weakness in translating research into concrete drug discovery and development, 
- the evolution of international regulations on access to genetic resources and the growing concern by stakeholders vis-à-vis the demands for patenting rights, evidence of safety, efficacy, good quality traditional medicinal products and a range of other ethical issues,

- the shortage of essential infrastructure in both the public (universities and other governmental institutions) and private sectors,

- the need for integrating and promoting the potential of medicinal plants as a source of health care. So far, there have been significant efforts within the framework of the Cameroon Ethnobotany Network and the Millennium Ecologic Museum, under the leadership of Late Professors Bernard-Aloys Nkongeneck, Daniel Lantum, Jacques Kamsu Kom and Jeanne Ngogang, towards the strengthening the capacity of Cameroonian traditional healers. Series of training were offered geared at improving their knowledge and practice on basic techniques of pharmaceutical sciences. Nowadays and more than ever, it is still an imperative to keep pace with the committments of these pioneering ethnobotanists and to continue adding efforts to boost research and development in the field of medicinal plants. As new and effective drugs are urgently needed, in the fight gainst COVID-19, research programs into alternative therapeutics including medicinal plants investigations need to be encouraged.

\section{Purpose of this review}

This review is part of the contribution of ethnobotany and ethnopharmacology sciences in the fight against COVID-19. It aims at providing a preliminary review of available literature on medicinal plants with potentials to be evaluated and developed for the management of COVID-19 in Cameroon.

The findings of this review will provide other researchers with opportunities to identify the right medicinal plants to be evaluated from a perspective of developing new drugs to combat COVID-19.

\section{Methodological approach}

\section{Theoretical framework to the selection of potential anti-COVID plants}

The theoretical framework for the study is based on a 3-step review approach.

First, we acknowledge that the use of medicinal plants for the treatment of viral infections in our traditional societies is ancient. Meanwhile, COVID-19 is a novel disease and consequently not yet known in our traditional knowledge system on diseases. However, evidence from existing literature supports the management of symptoms similar to those of COVID-19 using a diversity of plant-based recipes. A recent review by Poudel Adhikari et al. (2020) presented the most commonly reported symptoms of COVID-19. Those considered in this review were: fever/malaria, runny nose, cough, myalgia or fatigue, body pains and sore throat. This review is based on the assumption that a plant that has been used to manage at least 3 common symptoms of COVID-19 is a potential source of anti-COVID-19 molecules.

Secondly, the inhibitory effect of some secondary metabolites from medicinal plants on the 2019 novel corona virus protease have been reported by Zhang et al. (2020) in China, Mohammadi and Shaghaghi (2020) in Iran and Khaerunnisa et al. (2020) in Indonesia. In this regard, the identification of Cameroonian medicinal plants with potentials as anti-COVID-19 was based on the investigations of their phytochemical profile to select those that are source materials for these secondary metabolites. Besides the metabolites cited by the above-mentioned studies, alkaloids are also a rich source of active components of plants that have already been fruitfully developed into various chemotherapeutic compounds comprising Chloroquine, an antimalarial drug reported to be effective for the treatment of COVID-19 and many other viral infections (Moradi et al. 2017; Colson et al. 2020; Gao et al. 2020). The mechanism of the antiviral activity of alkaloids is based on the inhibition of replication of viruses. Hence, in this study, a plant known as an important source of alkaloid is also considered as potential anti-COVID-19. Similar bioactivity on 2019-nCov was also reported for hydrolysable tannins, natural polyphénols (Khalifa et al. 2020; Adem et al. 2020) and terpenoids (Shagaghi 2020). Therefore, we also consider of great potential for COVID-19 management, plants that are rich sources of these secondary metabolites.

Thirdly, the use of biologics that stimulate immune responses was suggested by Zumla et al. (2020) as a way to help patients resist the invading virus. There is an abundant literature reporting the use of plants by traditional medicine practitioners to boost the immune system in people living with HIV/AIDS (Anywar et al. 2020). In addition to the important role of boosting the immune system, evidence from the literature reveals the importance of antimalaria and antiviral drugs in the global therapeutics against COVID19 (Vellingiri et al. 2020). This is also the case for antiinflammatory drugs that may be effective against elevated levels of cytokines and useful in inhibiting viral infection. Hence, plants with immunostimulant, antiviral, anti-malaria and anti-inflammatory properties are considered in this study as of great potentials for COVID-19 management.

\section{Data collection and computation}

This review is based on data available in published literature. Bibliographic information on medicinal plants was 
searched in several databases including: Google Scholar, PubMed, Scopus, ScienceDirect, Researchgate, PROTA, GLOBEinMED, to retrieve all relevant papers. Key words used included among other, the symptoms of COVID-19 (fever/malaria, runny nose, cough, myalgia or fatigue, body pains and sore throat), immunostimulant, antiviral, antimalaria, anti-inflammatory, and secondary metabolites with confirmed inhibitory effect on the 2019 nCov (Allicin, Apigenin-7-glucoside, Catechin, Coumaroyltyramine, Curcumin, Desmethoxyreserpine, Diosmin, Epicatechin-gallate, Gingerol, Hesperidin, Kaempferol, Lignan, Luteolin-7-glucoside, Naringenin, Oleuropein, Pedunculagin, Punicalin, Quercetin, N-cis-feruloyltyramine, etc.).

A total of 119 papers were reviewed including books, journal articles, proceedings, preprints. The reference lists of some research articles were exploited to explore additional relevant studies. The database of the Global biodiversity Information Facility (GBIF) was searched to confirm the occurrences and distribution of the plant species recorded.

From the ethnobotanical and ethnomedical literature consulted, plants were selected and recorded based on their uses (focus on plants used to treat symptoms of COVID-19), their phytochemical composition (with a focus on plants rich in alkaloids, tannins, terpenoids and phenolics), their pharmacological activity (focused on plants with anti-inflammatory, immunomodulatory, antimalarial and anti-viral properties). All the plant species recorded were compiled in an Excel database.

The documented uses of each plant, the presence or absence of the targeted secondary metabolites and their documented pharmacological activity were used to generate a new data set which was analyzed by principal component analysis (PCA) to detect cluster formatting and the patterns of variability present in the data sets of the medicinal plant species recorded.

\section{Findings and implication}

\section{Confirmed anti-COVID19 molecules and their source plants in Cameroon}

The main protease (Mpro)/chymotrypsin like protease (3CLpro) from the 2019 novel corona virus, is reported to be a potential target for the inhibition of its replication ( $\mathrm{Lu}, 2020)$. Khaerunnisa et al. (2020) showed that luteolin-7-glucoside, demethoxycurcumin, apigenin-7-glucoside, oleuropein, curcumin, catechin, and epicatechin-gallate appeared to have the best potential to act as COVID-19 Mpro inhibitors. Faheem Khan et al. (2020) showed that epigallocatechin gallate (EGCG), a major constituent of green tea (Camelia sinensis), was the lead compound that could fit well into the binding sites of docked proteins of SARS-CoV-2 and recommended this molecule as a drug candidate for the treatment of COVID-19.

Mohammadi and Shaghaghi (2020) reported that secondary metabolites including kaempferol, quercetin, luteolin-7-glucoside, demethoxycurcumin, naringenin, apigenin-7-glucoside, oleuropein, curcumin, catechin, epicatechin-gallate, zingerol, gingerol, and allicin were potential inhibitor candidates for COVID-19 Mpro, with Curcumin showing the strongest interaction with the protease enzyme of COVID-19. A recent study by Zhang et al. (2020) has identified several Chinese medicinal plants classified as antiviral/antipneumonia-effective that directly inhibit the novel coronavirus, 2019-nCoV. The metabolites tested for this bioactivity were Betulinic acid, Coumaroyltyramine, Cryptotanshinone, Desmethoxyreserpine, Dihomo-c-linolenic acid, Dihydrotanshinone, Kaempferol, Lignan, Moupinamide, N-cis-feruloyltyramine, Quercetin, Sugiol, Tanshinone IIa.

Khalifa et al. (2020) showed that the Pedunculagin, tercatain, and punicalin, three hydrolysable tannins, successfully inhibit the protease enzyme of 2019 novel Corona Virus.

Adem et al. (2020) evaluated the efficacy of medicinal plant-based bioactive compounds against COVID-19 Mpro by a molecular docking study. They concluded that natural polyphénols including hesperidin, rutin, diosmin, apiin, diacetylcurcumin, (E)-1-(2-Hydroxy-4-methoxyphenyl)-3[3-[(E)-3-(2-hydroxy-4-methoxyphenyl)-3-oxoprop-1-enyl] phenyl]prop-2-en-1-one, and $\beta, \beta$ '-(4-Methoxy-1,3 phenylene)bis(2'-hydroxy-4',6'-dimethoxyacrylophenone were effective inhibitors of this new Corona Virus.

From the research conducted by these authors, it is clear that Cameroonian medicinal plants can provide source materials for these secondary metabolites. The review of the phytochemical screening done on Cameroonian medicinal plant species shows that 32 species native or naturalized in Cameroon are source materials for most of the above-mentioned secondary metabolites (Table 1). There is also evidence from available literature indicating diverse pharmacological properties for these species including antimicrobial, antiviral, analgesic, anti-inflammatry, antipyretic, antioxidant, and more. (Table 2). Besides Curcumine from turmeric (Curcuma loonga), some of those local plant species are interesting as they contain many of those active secondary metabolites. This is the case of Zanthoxyllum heitzii containing both Apigenin-7-glucoside and Oleuropein, and Citrus spp, a rich source of Diosmin, Lignan, Naringenin and Quercetin that showed high inhibitory effect on 2019 corona virus.

\section{Cameroonian medicinal plant used to manage symptoms of COVID 19}

The review yielded a total of 230 medicinal plants of potential for the management of COVID-19. From this general list 


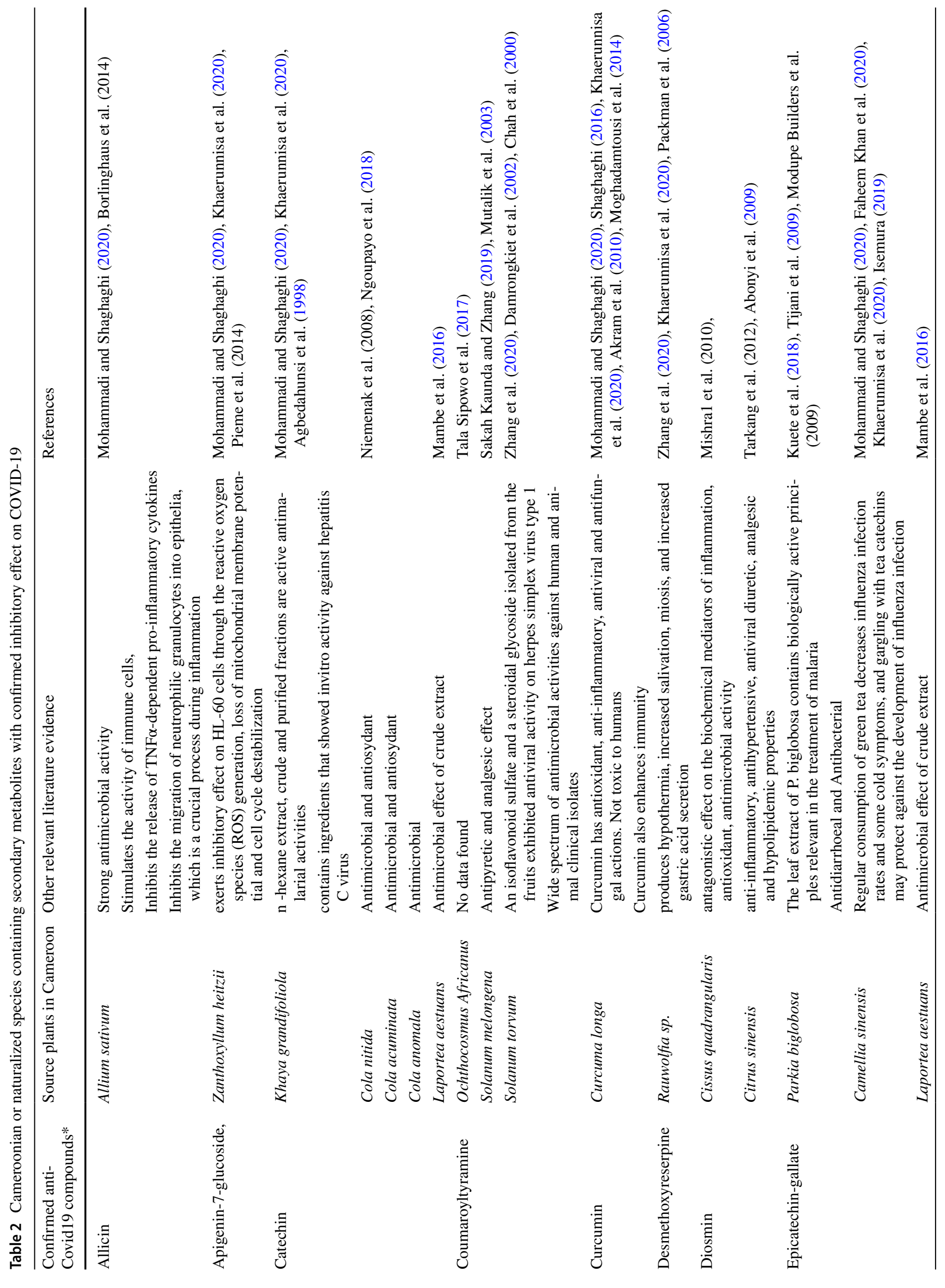




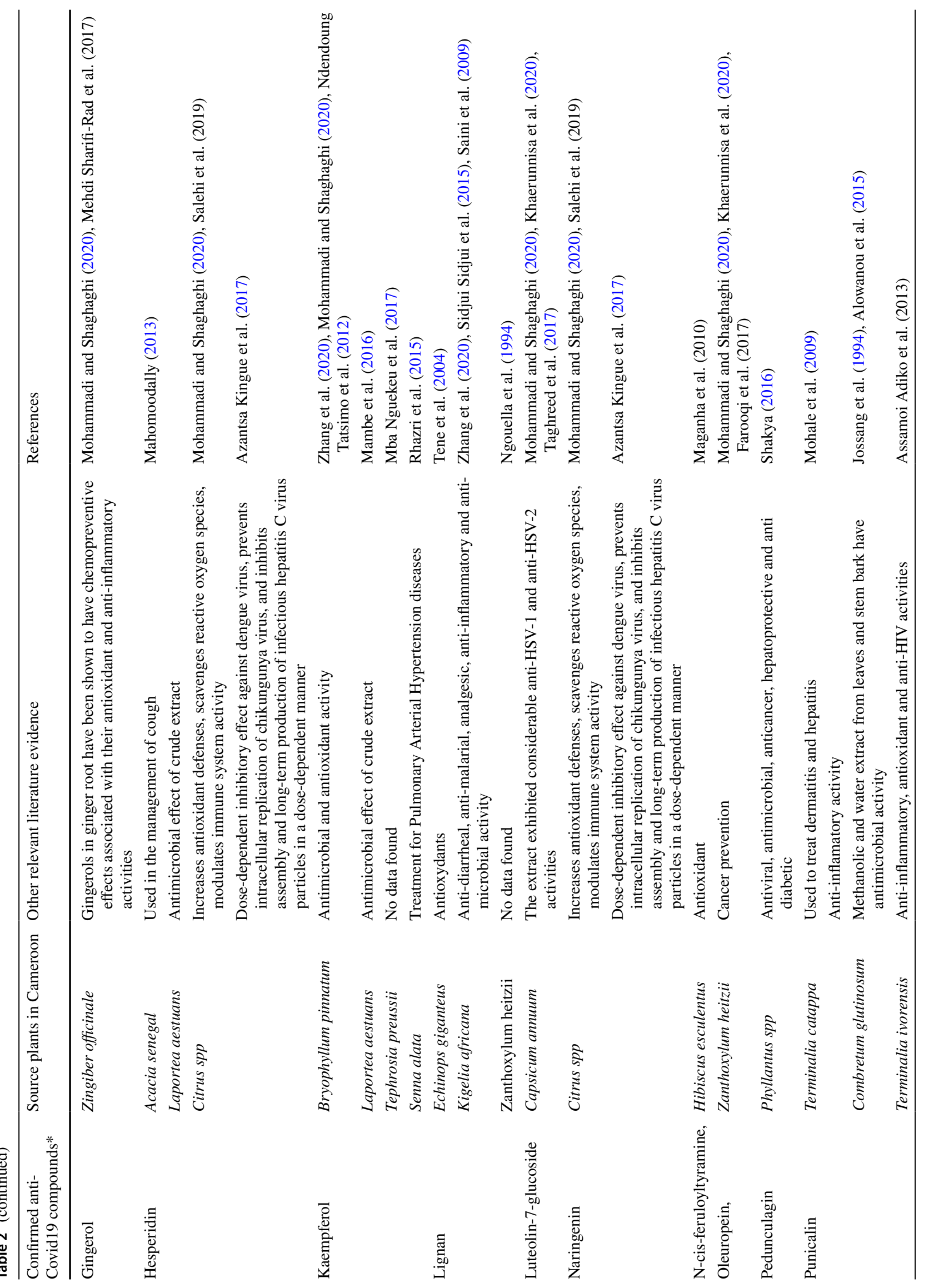


Table 3 Number of medicinal plants cited in the treatment of COVID-19 symptoms

\begin{tabular}{lll}
\hline Symptoms & $\begin{array}{l}\text { Number of plants } \\
\text { recorded }\end{array}$ & Percentage \\
\hline Catarrh/Runny nose & 20 & 3.8 \\
Cough & 138 & 26.3 \\
Fever & 241 & 45.9 \\
Myalgia/fatigue & 106 & 20.2 \\
Headache & 8 & 1.5 \\
Pains & 4 & 1.58 \\
Sore throat & 3 & 0.6 \\
\hline
\end{tabular}

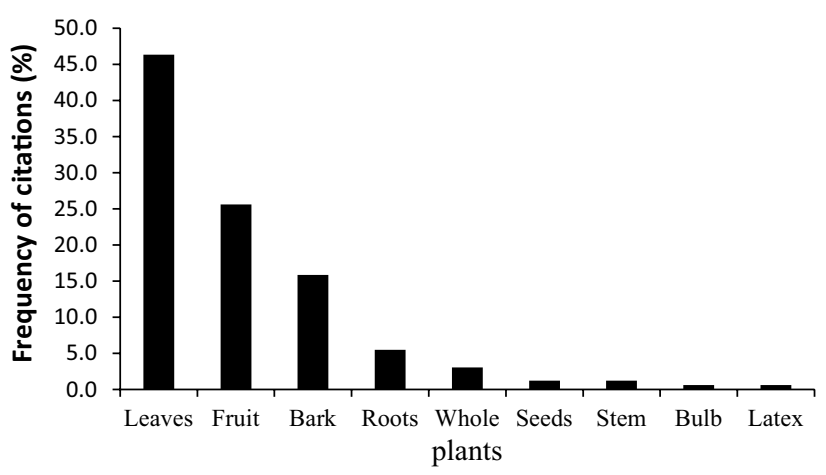

Fig. 1 Frequency of plant parts used in management of COVID symptoms

of plants recorded, 90 species were selected for being mentioned as used to manage at least 3 symptoms of COVID-19, and the remaining species were excluded (Table 4). These 90 species belong to 53 botanical families. The families with the greatest number of representatives are Rubiaceae ( 8 species), Asteraceae and Euphorbiaceae (6 species), Caesalpiniaceae and Meliaceae (5 species), Solanaceae (4 species), Apocynaceae, Combretaceae, Malvaceae, Sapotaceae and Verbenaceae (3 species).

The greatest number of citations was recorded for three of the six symptoms investigated: fever/malaria, cough and myalgia/fatigue (Table 3).

Various plant parts are used in the different treatments reported in the literature. However, leaves, fruits and bark were the most used parts, indicating that their utilization may not severely affect the sustainability of the resource base (Fig. 1).

Available data on the phytochemical screening of these selected species shows that the most distributed secondary metabolite in this selected sample of plants was alkaloids (36\%) (Fig. 2). Previous studies by Ntié-Kang et al. (2013) also confirmed the greater distribution of terpenoids (26\%), flavonoids (19.6\%) and alkaloids (11.2\%) in Cameroon's medicinal plants. 


\section{State of knowledge on Cameroonian medicinal plants with confirmed anti-inflammatory, anti-viral and immunostimulant properties}

Evidence from research conducted on SARS-COV and COVID-19 shows that the weakening of the immune system is one of the major contributing factors to the increased incidence of COVID complications like pneumonia and mortality in affected patients (Curbelo et al. 2017; TaghizadehHesary and Akbari 2020; Prompetchara et al. 2020). These authors argued that improving the immune system response may be effective in reducing the incidence of pneumonia, and reduction of inflammation may be effective in reducing the mortality rates due to pneumonia. From the literature data compiled, about 10 species have been documented for their beneficial effect in boosting the immune system. Among these species, 3 were also cited to treat at least 3 symptoms of COVID-19: Azadirachta indica and Momordica charantia and Vernonia amygdalina (Table 4). Of the total of 52 species documented for their anti-inflamatory activity, there are also 11 cited as used to treat COVID-19 symptoms. These are: Acanthus montanus, Eleusine indica, Entandrophragma cylindricum, Eremomastax speciosa, Erythrophleum suaveolens, Jatropha curcas, Kalanchoe crenata, Picralima nitida, Senna alata, Solanum torvum, Spathodea campanulata Vernonia amygdalina, and Vitellaria paradoxa (Table 4). A total of 14 species were cited for their antiviral properties on other virus-induced diseases, of which 5 are traditionally used to manage COVID-19 symptoms: Anickia chlorantha, Artemisia annua, Costus afer, Senna alata and Vernonia amygdalina (Table 5). A total of 78 species have been documented as used to treat malaria. Overall, the leaves, bark and roots are the most used plant parts as noted below (Fig. 3).

Overall, these species belongs to 53 different botanical families. The families with a higher number of representatives are Caesalpiniaceae (10 species), Asteraceae (3 species), Cucurbitaceae and Apocynaceae (3 species),

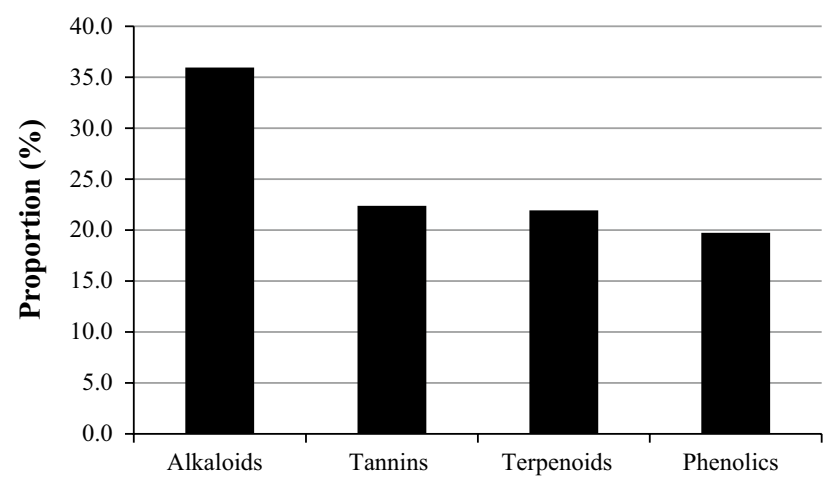

Fig. 2 Distribution of secondary metabolites in the recorded plants
Euphorbiaceae, Lamiaceae, Meliaceae, Acanthaceae, Combretaceae, Euphorbiaceae, Meliaceae and Mimosaceae (2 species).

\section{Summary and implication for the fight against COVID-19}

From this review, 230 Cameroonian medicinal plant species emerge as promising sources of ingredients for the fight against the 2019 novel corona virus. Among these species, about 32 contain secondary metabolites that have already been confirmed as anti-COVID-19 molecules. These are Abelmoschus esculentus, Acacia Senegal, Allium sativum, Bryophyllum pinnatum, Camellia sinensis, Capsicum annuum, Cissus quadrangularis, Citrus spp, Cola acuminata, $C$. anomala, C. nitida, Combretum glutinosum, Curcuma longa, Echinops giganteus, Khaya grandifoliola, Kigelia Africana, Laportea aestuans, Morinda morindoides, Ochthocosmus africanus, Parkia biglobosa, Phyllanthus spp, Rauwolfia sp., Senna alata, Solanum melongena, Solanum torvum, Tephrosia preussii, Terminalia catappa, Terminalia ivorensis, Zanthoxyllum heitzii and Zingiber officinale.

Of the 230 species recorded, 102 are already documented for their traditional use to manage at least 3 common symptoms of COVID-19. The PCA analysis separated 4 groups of medicinal plant species with axis 1 and 2 explaining $65.7 \%$ of the variability within the sample (Fig. 4).

The first group consists of plants treating at least three symptoms of COVID 19, containing key phytochemicals reported as being of interest for COVID management (alkaloids, phenolics, tannins and terpenoids) and having antimalaria properties. Representative species include Abelmoschus esculentus, Artemisia annua, Capsicum annun, Curcuma longa, Eucalyptus camaldulensis, Eremomastax speciosa, Kalenchoe crenata, Lippia multiflora, Morinda lucida, Senna alata, Solanum torvum, etc.

The second group consists of highly promising species like Azadirachta indica, Harungana madagascariensis, Mangifera indica, Momordica charantia, Picralima nitida, Trichilia emetica. This consists of plants used to treat COVID-19 symptoms which, at the same time are sources of the key phytochemicals and also have relevant pharmacological activities (antiviral, anti-inflammatory, immunostimulant, or containing secondary metabolites with confirmed anti-SARSCOV2 activity). Even when used alone, they can be evaluated and developed as potential remedies, while the other species may be used in association to each other for their complementary effects.

The third group consists of potential anti-malaria agents based on the species Allium sativum, Psidium guajava, Phyllanthus muellerianus, Occimum gratissimum, Stereospermom acuminatissimum, etc. 
Table 4 Cameroonian medicinal plant species used to manage at least 3 COVID 19 symptoms

\begin{tabular}{|c|c|c|c|c|c|}
\hline Species & Family & Part used* & Symptoms treated & Main phytochemicals** & Reference \\
\hline Abelmoschus esculentus & Sterculiaceae & $\mathrm{L}, \mathrm{Fr}$ & Cough, Fever, Myalgia & Tan, Phen, Terp & $\begin{array}{l}\text { Bogninou et al. (2018) } \\
\text { Tomar (2017), Alamgeer } \\
\text { Younis et al. (2018) }\end{array}$ \\
\hline Acanthus montanus & Acanthaceae & $\mathrm{L}$ & Cough, Fever, Myalgia & Alk & $\begin{array}{l}\text { Asongalem et al. (2004), } \\
\text { Kuete and Efferth } \\
\text { (2010), Etame et al. } \\
\text { (2018), Fongod et al. } \\
\text { (2013) }\end{array}$ \\
\hline Adansonia digitata & Bombacaceae & $\mathrm{Bk}$ & Cough, Fever, Myalgia & Alk & $\begin{array}{l}\text { Yinyang et al. (2014), } \\
\text { Arbonnier (2019), } \\
\text { Kamatou et al. (2011) }\end{array}$ \\
\hline Ageratum conyzoides & Asteraceae & $\mathrm{L}$ & Cough, Myalgia, fever & Alk, Terp & $\begin{array}{l}\text { Ming (1999), Jiofack et al. } \\
\text { (2008), Yinyang et al. } \\
\text { (2014) }\end{array}$ \\
\hline Alchornea cordifolia & Euphorbiaceae & $\mathrm{L}$ & Cough, Fever, Myalgia & Alk, Tan, Phen & $\begin{array}{l}\text { Ngaha et al. (2016), Ngou- } \\
\text { payo et al. (2018) }\end{array}$ \\
\hline Allium sativum & Liliaceae & Bulb & Cough, Fever, Myalgia & Alk & $\begin{array}{l}\text { Papu et al. (2014), Khoda- } \\
\text { dadi (2015) }\end{array}$ \\
\hline Aloe vera & Aloaceae & $\mathrm{L}$ & Cough, Fever, Myalgia & Alk & $\begin{array}{l}\text { Sahu et al. (2014), Yin- } \\
\text { yang et al. (2014) }\end{array}$ \\
\hline Alstonia boonei & Sapotaceae & $\mathrm{Bk}, \mathrm{Lx}, \mathrm{L}$ & Cough, Fever, Myalgia & Alk, Tan, Terp & $\begin{array}{c}\text { Jiofack et al. }(2008,2009) \text {, } \\
\text { Dibong et al. }(2015)\end{array}$ \\
\hline Amaranthus hybridus & Amaranthaceae & Wp & Cough, Fever, Myalgia & Alk, Terp & $\begin{array}{l}\text { Tinitana et al. (2016), } \\
\text { Etame et al. (2018) }\end{array}$ \\
\hline Ananas comosus & Annonaceae & Epc & Cough, Fever, Myalgia & Alk & $\begin{array}{l}\text { Hossain et al. (2015), } \\
\text { Yinyang et al. (2014) }\end{array}$ \\
\hline Anickia chloranta & Annonaceae & $\mathrm{Bk}$ & Cough, Fever, Myalgia & Alk, Phen, Tan & $\begin{array}{l}\text { Etame et al. (2018), } \\
\text { Njayou et al. (2008) }\end{array}$ \\
\hline Annona senegalensis & Annonaceae & $\mathrm{Bk}$ & Cough, Fever, Myalgia & Phen, Tan & $\begin{array}{l}\text { Tsabang et al. (2012), } \\
\text { Njayou et al. (2008) }\end{array}$ \\
\hline Annona muricata & Annonaceae & L, Fr, Se, Pulp & $\begin{array}{l}\text { Cough, Fever, pains, } \\
\text { catharrh }\end{array}$ & Alk, Tan & $\begin{array}{l}\text { Zorofchian Moghad- } \\
\text { amtousi et al. (2015), } \\
\text { Yinyang et al. (2014), } \\
\text { Tsobou et al. (2015) }\end{array}$ \\
\hline Anogeissus leiocarpus & Combretaceae & $\mathrm{Bk}$ & Cough, Fever, body pains & Tan & $\begin{array}{l}\text { Ahmad (2014), Ndjonka } \\
\text { et al. (2008) }\end{array}$ \\
\hline Anthocleista djalonensis & Loganiaceae & $\mathrm{Bk}$ & Cough, Fever, Myalgia & Alk, Phen, Tan & $\begin{array}{l}\text { Bassey et al. (2009), Leke } \\
\text { et al. (2012) }\end{array}$ \\
\hline Anthocleista nobilis & Loganiaceae & $\mathrm{Bk}$ & Cough, Fever, Myalgia & Alk, Phen, Tan, Ter & $\begin{array}{l}\text { Mosango (2007), Sima } \\
\text { et al. (2015) }\end{array}$ \\
\hline Artemisia аппиа & Asteraceae & $\mathrm{Wp}$ & Cough, Fever, Myalgia & Phen & $\begin{array}{l}\text { Jiofack et al. (2008), Iqbal } \\
\text { et al. (2012), Sadiq et al. } \\
\text { (2014) }\end{array}$ \\
\hline Azadirachta indica & Meliaceae & $\mathrm{Se}, \mathrm{L}, \mathrm{Bk}$ & Cough, Fever, Myalgia & Alk, Phen, Tan, Ter & $\begin{array}{l}\text { Jiofack et al. }(2009,2008) \text {, } \\
\text { Dash et al. (2017) }\end{array}$ \\
\hline Brassica oleracea & Brassicaceae & $\mathrm{L}$ & $\begin{array}{l}\text { Cough, Fever, Myalgia } \\
\text { Sore throat }\end{array}$ & Alk & Yinyang et al. (2014) \\
\hline Bridelia ferruginea & Euphorbiaceae & $\mathrm{Bk}$ & Cough, Fever, Myalgia & Alk, Tan, Terp & $\begin{array}{l}\text { Ndam et al. (2014), } \\
\text { Jose and Kayode (2009), } \\
\text { Olumayokun et al. } \\
\text { (2012) }\end{array}$ \\
\hline Bridelia micrantha & Euphorbiaceae & $\mathrm{Bk}$ & Cough, Fever, Myalgia & Alk, Phen, Tan, Ter & $\begin{array}{l}\text { Arbonnier (2019), Etono } \\
\text { et al. (2019), Maroyi } \\
\text { (2017) }\end{array}$ \\
\hline Camellia sinensis & Theaceae & $\mathrm{L}$ & Cough, Fever, Myalgia & Alk & $\begin{array}{l}\text { Yinyang et al. (2014), } \\
\text { Namukobea et al. (2011), } \\
\text { Sharangi (2009) }\end{array}$ \\
\hline
\end{tabular}


Table 4 (continued)

\begin{tabular}{|c|c|c|c|c|c|}
\hline Species & Family & Part used* & Symptoms treated & Main phytochemicals $* *$ & Reference \\
\hline Capsicum аппиит & Solanaceae & L, Fr & $\begin{array}{l}\text { Cough, Headache, } \\
\text { Myalgia }\end{array}$ & Alk & $\begin{array}{l}\text { Salehia et al. (2018), Yin- } \\
\text { yang et al. (2014) }\end{array}$ \\
\hline Capsicum frutescens & Solanaceae & $\mathrm{L}, \mathrm{Fr}$ & $\begin{array}{l}\text { Cough, Headache, } \\
\text { Myalgia }\end{array}$ & Alk, Terp & $\begin{array}{l}\text { Salehia et al. (2018), Nou- } \\
\text { medem et al. (2013) }\end{array}$ \\
\hline Carica papaya & Cacicaceae & L, Fr & Cough, Fever, Myalgia & Alk, Tan, Terp & $\begin{array}{l}\text { SIvarajah (2015), Sebua } \\
\text { and Maroyi (2013) }\end{array}$ \\
\hline Catharanthus roseus & Apocynaceae & $\mathrm{L}$ & $\begin{array}{l}\text { Cough, Sorethroat, } \\
\text { Myalgia }\end{array}$ & Alk & $\begin{array}{l}\text { Das and Sharangi (2017), } \\
\text { Yinyang et al. (2014) }\end{array}$ \\
\hline Chromolaena odorata & Asteraceae & $\mathrm{L}$ & Cough, Fever, Myalgia & Alk, Tan, Terp & $\begin{array}{l}\text { Vaisakh et Pandey (2012), } \\
\text { Tamo et al. (2016), }\end{array}$ \\
\hline Cinchona calisaya & Rubiaceae & B, Rt, L, Fr & Cough, Fever, Myalgia & Alk & $\begin{array}{l}\text { Eyal (2018), Yinyang et al. } \\
\text { (2014) }\end{array}$ \\
\hline Cinchona officinalis & Rubiaceae & B, Rt, L, Fr & Cough, Fever, Myalgia & Alk & $\begin{array}{l}\text { Eyal (2018), Yinyang et al. } \\
\text { (2014) }\end{array}$ \\
\hline Cinchona pubescens & Rubiaceae & B, Rt, L, Fr & Cough, Fever, Myalgia & Alk & $\begin{array}{l}\text { Eyal (2018), Yinyang et al. } \\
\text { (2014) }\end{array}$ \\
\hline Citrus aurantifolia & Rutaceae & $\mathrm{L}, \mathrm{Fr}$ & $\begin{array}{l}\text { Headache, colds, coughs, } \\
\text { sore throats }\end{array}$ & Alk & $\begin{array}{l}\text { Enejoh et al. (2015), Yin- } \\
\text { yang et al. (2014) }\end{array}$ \\
\hline $\begin{array}{l}\text { Cochlospermum plan- } \\
\text { chonii }\end{array}$ & Cochlospermaceae & $\mathrm{L}, \mathrm{Fr}$ & Cough, Fever, Myalgia & Alk, Phen, Tan, Terp & $\begin{array}{l}\text { Isah et al. (2013), Usman } \\
\text { et al. (2013), Mamidou } \\
\text { Koné et al. (2005) }\end{array}$ \\
\hline Cola acuminata & Malvaceae & $\mathrm{L}, \mathrm{Fr}$ & Cough, Fever, Myalgia & Alk, Phen & $\begin{array}{l}\text { Otoide and Olanipekun } \\
\text { (2018), Tchuenguem } \\
\text { et al. (2017), Yinyang } \\
\text { et al. (2014), Lowe et al. } \\
\text { (2014) }\end{array}$ \\
\hline Cola nitida & Malvaceae & $\mathrm{L}, \mathrm{Fr}$ & $\begin{array}{l}\text { Headache, Fever, } \\
\text { Myalgia }\end{array}$ & Alk & $\begin{array}{l}\text { Olukayode et al. (2017), } \\
\text { Yinyang et al. (2014) }\end{array}$ \\
\hline Combretum micranthum & Combretaceae & $\mathrm{L}$ & $\begin{array}{l}\text { Catarrh, Cough, Fever, } \\
\text { Myalgia }\end{array}$ & Alk, Terp & $\begin{array}{l}\text { Welch (2010), Chinsembu } \\
\text { and Hedimbi (2010), } \\
\text { Yinyang et al. (2014), } \\
\text { Dawe et al. (2013) }\end{array}$ \\
\hline Costus afer & Costaceae & St & $\begin{array}{l}\text { Catarrh, Cough, Fever, } \\
\text { Myalgia }\end{array}$ & Phen & $\begin{array}{l}\text { Boison et al. (2019), Tch- } \\
\text { uenguem et al. (2017) }\end{array}$ \\
\hline Crossopteryx febrifuga & Rubiaceae & L, Fr & Cough, Fever, Myalgia & Alk & $\begin{array}{l}\text { Salawu et al. (2008), } \\
\text { Arbonier (2019), Maiga } \\
\text { et al. (2006) }\end{array}$ \\
\hline Curcuma longa & Zingiberaceae & $\mathrm{Rz}$ & Cough, Fever, Myalgia & Terp & $\begin{array}{l}\text { Velayudhan et al. (2012), } \\
\text { Gardini et al. (2009) }\end{array}$ \\
\hline Cymbopogon citratus & Poaceae & $\mathrm{L}$ & $\begin{array}{l}\text { Cough, Fever, Headache, } \\
\text { Sore throat, Myalgia }\end{array}$ & Alk, Terp & $\begin{array}{l}\text { Etame et al. (2018), Yem- } \\
\text { ele et al. (2014), Yinyang } \\
\text { et al. (2014), Shah et al. } \\
\text { (2011) }\end{array}$ \\
\hline Diospyros mespiliformis & Ebenaceae & $\mathrm{L}, \mathrm{Fr}$ & Cough, Fever, Myalgia & Alk, Phen, Tan & $\begin{array}{l}\text { Hegazy et al. (2019), } \\
\text { Ahmed and Mahmud } \\
(2017)\end{array}$ \\
\hline Dissotis rotundifolia & Melastomataceae & $\mathrm{L}$ & $\begin{array}{l}\text { Catarrh, Cough, Fever, } \\
\text { Myalgia }\end{array}$ & Alk, Phen, Tan & $\begin{array}{l}\text { Jiofack et al. (2009), } \\
\text { Yinyang et al. (2014), } \\
\text { Yeboah and Osafo } \\
(2017)\end{array}$ \\
\hline Eleusine indica & Poaceae & $\mathrm{Wp}$ & Cough, Fever, Myalgia & Alk & $\begin{array}{l}\text { Etame et al. (2018), Patta- } \\
\text { nayak and Maity (2017), } \\
\text { Sagnia et al. (2014), } \\
\text { Jiofack et al. (2008), }\end{array}$ \\
\hline Emilia coccinea & Asteraceae & $\mathrm{Wp}$ & Cough, Fever, Myalgia & Alk, Tan, Terp & $\begin{array}{l}\text { Nwachukwu et al. (2017), } \\
\text { Tsobou et al. (2015), }\end{array}$ \\
\hline
\end{tabular}


Table 4 (continued)

\begin{tabular}{|c|c|c|c|c|c|}
\hline Species & Family & Part used* & Symptoms treated & Main phytochemicals $* *$ & Reference \\
\hline Eremomastax speciosa & Acanthaceae & $\mathrm{L}$ & $\begin{array}{l}\text { Catarrh, Cough, Fever, } \\
\text { Myalgia, Pains }\end{array}$ & Alk, Tan, Terp & $\begin{array}{l}\text { Jiofack et al. (2008), Tso- } \\
\text { bou et al. (2015), Sagnia } \\
\text { et al. (2014) }\end{array}$ \\
\hline $\begin{array}{l}\text { Eucalyptus camaldu- } \\
\text { lensis }\end{array}$ & Myrtaceae & $\mathrm{L}, \mathrm{Fr}, \mathrm{Bk}, \mathrm{Rt}$ & Cough, Fever, Myalgia & Alk, Tan, Phen, Terp & $\begin{array}{l}\text { Jiofack et al. (2008), Sani } \\
\text { et al. (2014) }\end{array}$ \\
\hline Euphorbia hirta & Euphorbiaceae & $\mathrm{Wp}$ & Cough, Fever, Myalgia & Alk, Tan, Terp & $\begin{array}{l}\text { Tamo et al. (2016), Kumar } \\
\text { et al. (2010) }\end{array}$ \\
\hline Eurycoma longifolia & Simaroubaceae & L, Fr & Cough, Fever, Myalgia & Alk, Terp & $\begin{array}{l}\text { Norhidayah Mohamed } \\
\text { et al. (2015), Mohamad } \\
\text { et al. (2010) }\end{array}$ \\
\hline Faidherbia albida & Mimosaceae & $\mathrm{Bk}$ & Cough, Catarrh, Fever & Alk, Tan, Terp, Phen & $\begin{array}{l}\text { Ismail et al. (2014), } \\
\text { Arbonier (2019), Marwa } \\
\text { et al. (2018) }\end{array}$ \\
\hline Garcinia cola & Clusiaceae & Fr & Cough, Fever, Myalgia & Alk & $\begin{array}{l}\text { Jiofack et al. (2008, 2009), } \\
\text { Betti (2002), Yinyang } \\
\text { et al. (2014) }\end{array}$ \\
\hline Guiera senegalensis & Combretaceae & $\mathrm{L}$ & Cough, Fever, Myalgia & Alk, Tan, Phen, Terp & $\begin{array}{l}\text { Shafei et al. (2016), } \\
\text { Arbonier (2019), Som- } \\
\text { boro et al. (2011) }\end{array}$ \\
\hline $\begin{array}{l}\text { Harungana madagas- } \\
\text { cariensis }\end{array}$ & Hypericaceae & $\mathrm{Bk}$ & Cough, Fever, Myalgia & Alk, Phen & $\begin{array}{l}\text { Nimenibo-Uadia and Nwa- } \\
\text { chukwu (2017), Ndam } \\
\text { et al. (2014) }\end{array}$ \\
\hline Hibiscus sabdarifa & Malvaceae & $\mathrm{L}$ & Cough, Fever, Myalgia & Alk & $\begin{array}{l}\text { Suresh and Ammaan } \\
\text { (2017), Yinyang et al. } \\
\text { (2014) }\end{array}$ \\
\hline Holarrhena floribunda & Apocynaceae & $\mathrm{Bk}, \mathrm{L}$ & Cough, Fever, Myalgia & Alk & $\begin{array}{l}\text { Hoekou et al. (2017), } \\
\text { Yinyang et al. (2014) }\end{array}$ \\
\hline Hoslundia opposita & Lamiaceae & Rt & $\begin{array}{l}\text { Cough, Fever, Sore } \\
\text { throat }\end{array}$ & Phen, Tan & $\begin{array}{l}\text { Arbonier (2019), Sadri } \\
\text { (2017), Ndjonka et al. } \\
(2008)\end{array}$ \\
\hline Hymenocardia acida & Euphorbiaceae & $\mathrm{L}, \mathrm{Rt}$ & Cough, Fever, Myalgia & Terp & $\begin{array}{l}\text { Amoa Onguéné et al. } \\
\text { (2013), Tor-Anyiin Ter- } \\
\text { rumun et al. (2013) }\end{array}$ \\
\hline Jatropha curcas & Euphorbiaceae & $\mathrm{L}$ & Cough, Fever, Headache & Alk, Phen & $\begin{array}{l}\text { Arbonier (2019), Abdel- } \\
\text { gadir and Van Staden } \\
\text { (2013), Oskoueian et al. } \\
\text { (2011) }\end{array}$ \\
\hline Kalenchoe crenata & Crassulacées & $\mathrm{L}$ & $\begin{array}{l}\text { Cough, Fever, Headache, } \\
\text { Myalgia }\end{array}$ & Alk, Terp & $\begin{array}{l}\text { Yinyang et al. (2014), } \\
\text { Jiofack et al. (2008), } \\
\text { Nguelefack et al. (2006) }\end{array}$ \\
\hline Khaya senegalensis & Meliaceae & $\mathrm{L}, \mathrm{Fr}$ & Cough, Fever, Myalgia & Alk, Phen, Tan, Terp & $\begin{array}{l}\text { Chukwudi Ugoh et al. } \\
\text { (2014), Arbonier (2019), } \\
\text { Makut et al. (2008) }\end{array}$ \\
\hline Lantana camara & Verbenaceae & $\mathrm{L}$ & Cough, Fever, Catarrh & Alk, Tan, Terp & $\begin{array}{l}\text { Tsobou et al. (2015), } \\
\text { Kalita et al. (2012) }\end{array}$ \\
\hline Lippia multiflora & Verbenaceae & $\mathrm{L}$ & Cough, Fever, Catarrh & Tan, Terp & $\begin{array}{c}\text { Gandonou et al. (2017), } \\
\text { Djengue et al. (2017) }\end{array}$ \\
\hline Mangifera indica & Anacardiaceae & $\mathrm{Bk}$ & Cough, Fever, Catarrh & Alk, Terp & $\begin{array}{l}\text { Mahalik et al. (2020), } \\
\text { Yemele et al. (2014), } \\
\text { Yinyang et al. (2014) }\end{array}$ \\
\hline Maytenus senegalensis & Celastraceae & $\mathrm{L}$ & Catarrh, Cough, Fever & Phen, Tan & $\begin{array}{l}\text { Arbonier (2019), Zangueu } \\
\text { et al. (2018), Veloso } \\
\text { et al. (2017) }\end{array}$ \\
\hline Melissa officinalis & Lamiaceae & $\mathrm{L}, \mathrm{Fr}$ & Catarrh, Cough, Fever & Alk & $\begin{array}{l}\text { Miraj et al. (2017), Yin- } \\
\text { yang et al. (2014) }\end{array}$ \\
\hline
\end{tabular}


Table 4 (continued)

\begin{tabular}{|c|c|c|c|c|c|}
\hline Species & Family & Part used* & Symptoms treated & Main phytochemicals** & Reference \\
\hline Milicia excelsa & Moraceae & $\mathrm{Bk}$ & Catarrh, Cough, Fever & Alk, Phen, Tan & $\begin{array}{l}\text { Jiofack et al. (2008), Betti } \\
\text { (2002), Akinpelu et al. } \\
(2020)\end{array}$ \\
\hline Mitragyna inermis & Rubiaceae & $\mathrm{L}, \mathrm{Bk}, \mathrm{Rbk}$ & Catarrh, Cough, Fever & Alk, Phen, Tan, Terp & $\begin{array}{l}\text { Mahougnan Toklo et al. } \\
\text { (2020), Arbonier (2019), } \\
\text { Konkon et al. (2008) }\end{array}$ \\
\hline Momordica charantia & Cucurbitaceae & $\mathrm{L}$ & Cough, Fever, Pains & Alk, Phen, Tan, Terp & $\begin{array}{l}\text { Jiofack et al. (2009), } \\
\text { Mozaniel et al. (2018) }\end{array}$ \\
\hline Morinda lucida & Rubiaceae & L, Fr & Cough, Fever, Pains & Tan & $\begin{array}{l}\text { Adeleye and Ajamu } \\
\text { (2018), Ndam et al. } \\
(2014)\end{array}$ \\
\hline Myristica fragrans & Myristicaceae & $\mathrm{L}, \mathrm{Fr}$ & Catarrh, Fever, Myalgia & Alk, Phen,Terp, & $\begin{array}{l}\text { Asgarpanah and } \\
\text { Kazemivash (2012), } \\
\text { Bamidele et al. (2011) }\end{array}$ \\
\hline Olax subscorpioidea & Olacaceae & $\mathrm{L}, \mathrm{Fr}$ & Cough, Fever, Myalgia & Alk, Phen, Tan, Terp & $\begin{array}{l}\text { Osuntokun and Omolola } \\
\text { (2019), Banjo et al. } \\
\text { (2018), }\end{array}$ \\
\hline Paullinia pinnata & Spindaceea & $\mathrm{L}, \mathrm{Rt}$ & Cough, Fever, Myalgia & Alk, Tan, Ph & $\begin{array}{l}\text { Tsobou et al. (2015), } \\
\text { MAriame et al.(2016), } \\
\text { Arbonier (2019), Ariyo } \\
\text { et al. (2020) }\end{array}$ \\
\hline Pavetta crassipes & Rubiaceae & $\mathrm{L}, \mathrm{Fr}$ & Cough, Fever, Myalgia & Alk, Phen & $\begin{array}{l}\text { Katsayal and Danfodiyo } \\
\text { (2002), Arbonier (2019), } \\
\text { Bello et al. (2011) }\end{array}$ \\
\hline Picralima nitida & Apocynaceae & Fr, Rt & Cough, Fever, Myalgia & Alk, Tan, Terp & $\begin{array}{l}\text { Tamo et al. (2016), Tsobou } \\
\text { et al. (2015), Erharuyi1 } \\
\text { et al. (2014) }\end{array}$ \\
\hline Piliostigma thonningii & Caesalpiniaceae & $\mathrm{L}, \mathrm{Fr}$ & $\begin{array}{l}\text { Cough, Fever, Sore } \\
\text { Throat }\end{array}$ & Phen, Tan & $\begin{array}{l}\text { Afolayan et al. (2018), } \\
\text { Kazhila (2016), Njayou } \\
\text { et al. (2008) }\end{array}$ \\
\hline Sarcocephalus latifolius & Rubiaceae & $\mathrm{Bk}, \mathrm{L}, \mathrm{Fr}$ & Cough, Fever, Myalgia & Ter, Phen & $\begin{array}{l}\text { Arbonier (2019), Yesufu } \\
\text { et al. (2014), Kaboré } \\
\text { et al. (2014) }\end{array}$ \\
\hline Senna alata & Caesalpiniaceae & $\mathrm{L}, \mathrm{Fr}$ & Cough, Fever, Myalgia & Alk, Tan & Tsobou et al. (2015) \\
\hline Senna occidentalis & Caesalpiniaceae & $\mathrm{L}, \mathrm{Fr}$ & Cough, Fever, Myalgia & Alk, Phen, Tan, Terp & $\begin{array}{l}\text { Singh et al. (2019), Musa } \\
\text { et al. (2018) }\end{array}$ \\
\hline Senna sieberiana & Caesalpiniaceae & $\mathrm{L}, \mathrm{Fr}$ & $\begin{array}{l}\text { Headache, Fever, } \\
\text { Myalgia }\end{array}$ & Alk, Phen, Tan & $\begin{array}{l}\text { Archer et al. (2019), } \\
\text { Archer et al. (2019) }\end{array}$ \\
\hline Solanum nigrum & Solanaceae & $\mathrm{L}, \mathrm{Fr}$ & Cough, Fever, Myalgia & Alk, Terp & $\begin{array}{l}\text { Yinyang et al. (2014), } \\
\text { Noumedem et al. (2013), } \\
\text { Ramya et al. (2011) }\end{array}$ \\
\hline Solanum torvum & Solanaceae & $\mathrm{L}$ & Cough, Fever, Myalgia & Alk, Phen, Tan & $\begin{array}{l}\text { Kannan et al. (2012), } \\
\text { Jaiswal (2012), Kuete } \\
\text { and Efferth (2010), }\end{array}$ \\
\hline Spathodea campanulata & Bignoniaceae & $\mathrm{Bk}, \mathrm{L}$ & Cough, Fever, Myalgia & Terp & Yemele et al. (2014) \\
\hline Terminalia laxiflora & Combretaceae & $\mathrm{L}, \mathrm{Fr}$ & Cough, Fever, Myalgia & Alk, Phen, Tan, Terp & $\begin{array}{l}\text { Salih et al. (2018), Salih } \\
\text { et al. (2017) }\end{array}$ \\
\hline Trema orientalis & Ulmaceae & $\mathrm{L}, \mathrm{Fr}$ & Cough, Fever, Myalgia & Alk, Phen, Tan, Ter & $\begin{array}{l}\text { Akin et al. (2016), Adi- } \\
\text { nortey et al. (2013) }\end{array}$ \\
\hline Trichilia emetica & Meliaceae & $\mathrm{Bk}, \mathrm{L}$ & Cough, Fever, Myalgia & Alk, Phen, Tan, Terp & $\begin{array}{l}\text { Arbonier (2019), Kouit- } \\
\text { cheu et al. (2017), Diarra } \\
\text { et al. (2015), Šutovská } \\
\text { et al. (2009) }\end{array}$ \\
\hline Vernonia amygdalina & Asteraceae & $\mathrm{L}$ & Cough, Fever, Myalgia & Terp & $\begin{array}{l}\text { Fongnzossie et al. (2017), } \\
\text { Yeep et al. (2010) }\end{array}$ \\
\hline Vernonia colorata & Asteraceae & $\mathrm{L}$ & Cough, Fever, Myalgia & Terp & $\begin{array}{l}\text { Tsobou et al. (2015), Cioffi } \\
\text { et al. (2014) }\end{array}$ \\
\hline
\end{tabular}


Table 4 (continued)

\begin{tabular}{|c|c|c|c|c|c|}
\hline Species & Family & Part used* & Symptoms treated & Main phytochemicals** & Reference \\
\hline Vitellaria paradoxa & Sapotaceae & $\mathrm{Bk}, \mathrm{Fr}$ & Cough, Fever, Myalgia & Terp & $\begin{array}{l}\text { Mbaveng et al. (2011), } \\
\text { Maanikuu and Peker } \\
\text { (2017), Israel (2014), } \\
\text { Fongnzossie et al. (2017) }\end{array}$ \\
\hline Vitex simplicifolia & Verbenaceae & $\mathrm{L}$ & Cough, Fever, Myalgia & Alk, Phen, Tan, Terp & $\begin{array}{l}\text { Arbonier (2019) Salim and } \\
\text { Dikko (2016), Salim and } \\
\text { Imam (2016) }\end{array}$ \\
\hline Ximenia americana & Olacaceae & $\mathrm{L}, \mathrm{Fr}$ & Cough, Fever, Myalgia & Phen, Tan, Terp & $\begin{array}{l}\text { Urso et al. (2013), Hunde } \\
\text { Feyssa et al. (2012), } \\
\text { Monte et al. (2012) }\end{array}$ \\
\hline
\end{tabular}

* $L$ leave, $B k$ bark, $S t$ Stem, $R t$ roots, $F r$ fruit, $S e$ seed, $F l$ flower, $T b k$ root bark, $W p$ whole plant, $L x$ latex

**Alk alkaloids, Tan tannins, Terp terpenoids, $P h$ Phenolics

The fourth group consists of immunostimulants, antiinflamatory, antiviral agents and plants containing some secondary metabolites with confirmed anti-COVID-19 properties, with representative species like Moringa oleifera, Panda oleosa, Tapinanthus globuliferus, Zanthoxyllum heitzii, and Vernonia amygdalina.

Overall, the recorded medicinal plant species offers an array of possibility of using individual species or combinations of species for their complementary effects, based on the clinical symptoms showed by the patients and the therapeutic objective to be achieved.

\section{Challenges and way forward}

In developing countries with poor access to health facilities like Cameroon, medicinal plants are the richest and most available sources for use and even for drug discovery. In such situations when our societies are desperate to discover cures for new and deadly disease like COVID-19, the contribution of herbal medicine in early response strategies should be promoted. Though the country's pharmaceutical potentials are immense, constraints and challenges however exist at all levels. To effectively address these shortcomings, a strong political-will and support of the Cameroonian government will be crucial.

\section{Research and development}

Research in ethnobotany, ethnopharmacology and bioactive components of medicinal plants of Cameroon has been ongoing for quite some time by University laboratories, by the Institute of Medical Research and Medicinal Plants Studies (IMPM) and by independent researchers. However, a systematic and concerted approach to this activity has been lacking. Much of this research has been academic and the concept of applied research in plant-based drug development has not received much attention. Although enough has been done in propagation of medicinal plants, research in support of industrial development, appropriate processing technologies to improve quality and yield, new formulations to new products and the marketing of finished products is still poorly developed. Actually, many medicinal plants sourced from Cameroon were involved in patents, most of which are owned by foreign entities (Oldham et al. 2013).

Capacity building and financial support are a necessity at all level in order to stimulate active research on natural medicinal products at the local level. Specifically, efforts have to be geared towards developing and sponsoring applied research on natural products and drug discovery. It is indeed paradoxical that with the country's medicinal plant potentials, herbal drug discovery has not yet reached the expected performance.

\section{Capacity building}

One of the main problems facing the use of herbal medicines is the proof requirement of their usefulnes, safety and effectiveness. Unfortunately, research and training activities for traditional medicine practitioners have not received due support and attention. As a result, the quantity, quality, safety and efficacy of herbal preparations are far from sufficient to meet demand. These weaknesses could be corrected by capacity building and low-cost technologies for the industrial production of traditional medicines to make them more effective, stable, reproducible, controlled, and in galenic forms that can easily be transported. Capacity building will be vital for also organizing the stakeholders and integrating their practices into the perspectives of modern research and development continuum. By so doing, the indiscriminate sale and advertisement of herbal products in all forms of media without compliance to the existing regulations would be discouraged. 
Table 5 Recorded plants documented for their anti-inflammatory, anti-viral and/or immunostimulant properties

\begin{tabular}{|c|c|c|c|c|}
\hline Scientific name & Family & Part used & $\begin{array}{l}\text { Existing pharmacological } \\
\text { records }\end{array}$ & References \\
\hline Acacia polyacantha & Fabaceae & Leaves & Antimalaria & $\begin{array}{l}\text { Bashige-Chiribagula et al. } \\
\text { (2017) }\end{array}$ \\
\hline Acanthus montanus & Acanthaceae & $\mathrm{L}$ & Anti-inflammatory & Kuete and Efferth (2010) \\
\hline Adenocarpus mannii & Caesalpiniaceae & $\mathrm{L}$ & $\begin{array}{l}\text { Methanol extracts pos- } \\
\text { sess immunomodulatory } \\
\text { activity }\end{array}$ & Kuate (2015) \\
\hline Aframomum citratum & Zingiberaceae & Fruit & Antimalaria & Tane et al. (2005) \\
\hline Aframomum latifolium & Zingiberaceae & Fruit & Antimalaria & Tane et al. (2005) \\
\hline Aframomит melegueta & Zingiberaceae & Seeds & Antimalaria & Tane et al. (2005) \\
\hline Aframomum sceptrum & Zingiberaceae & Fruit & Antimalaria & Tane et al. (2005) \\
\hline Aframoтит zambesiacum & Zingiberaceae & Fruit & Antimalaria & Tane et al. (2005) \\
\hline Ageratum conyzoides & Asteraceae & $\mathrm{L}$ & Antimalaria & $\begin{array}{l}\text { Jiofack et al. (2008), Yinyang } \\
\text { et al. (2014) }\end{array}$ \\
\hline Albizia adiantifolia & Mimosaceae & Leaves & Antimalaria & $\begin{array}{l}\text { Bashige-Chiribagula et al. } \\
\text { (2017) }\end{array}$ \\
\hline Albizia zygia & Mimosaceae & $\mathrm{L}, \mathrm{Rt}$ & Anti-inflammatory & Asante-Kwatia et al. (2020) \\
\hline Albizia zygia & Mimosaceae & Leaf & Antimalaria & Titanji et al. (2008) \\
\hline Alchemilla kiwuensis & Rosaceae & $\mathrm{Wp}$ & Anti-inflammatory & Kamtchueng et al. (2017) \\
\hline Alchornea cordifolia & Euphorbiaceae & $\mathrm{L}$ & Antimalaria & Ngoupayo et al. (2015) \\
\hline Allanblackia monticola & Clusiaceae & $\mathrm{Bk}$ & Anti-inflammatory & Kuete and Efferth (2010) \\
\hline Allanblackia monticola & Clusiaceae & Stem bark & Antimalaria & Titanji et al. (2008) \\
\hline Allium sativum & Liliaceae & $\mathrm{Bu}$ & $\begin{array}{l}\text { Anti-inflammatory, anti- } \\
\text { oxidant, bronchitis, chronic } \\
\text { fever }\end{array}$ & Khodadadi (2015) \\
\hline Allium sativum & Liliaceae & Bulb & Antimalaria & Khodadadi (2015) \\
\hline Alstonia boonei & Sapotaceae & Bk, Lx, L & Antimalaria & $\begin{array}{l}\text { Jiofack et al. }(2008,2009) \text {, } \\
\text { Dibong et al. }(2015)\end{array}$ \\
\hline Anisophyllea pomisofera & Rhizophoraceae & Leaves & Antimalaria & $\begin{array}{l}\text { Bashige-Chiribagula et al. } \\
\text { (2017) }\end{array}$ \\
\hline Annickia chlorantha & Anonaceae & $\mathrm{Bk}$ & $\begin{array}{l}\text { Antiviral (Hepatitis A,B; C } \\
\text { and D) }\end{array}$ & Ngono Ngane et al. (2011) \\
\hline Annona muricata & Annonaceae & L, Fr, Se, Pulp & Antimalaria & $\begin{array}{l}\text { Yinyang et al. (2014), Tsobou } \\
\text { et al. (2015) }\end{array}$ \\
\hline Anogeissus leiocarpus & Combretaceae & Leaves & Antimalaria & Ahmad (2014) \\
\hline Anonidium mannii & Anonaceae & $\mathrm{Bk}$ & Antiinflammatory & Mokale Kognou et al. (2020) \\
\hline Anopyxis klaineana & Rhizophoraceae & $\mathrm{Bk}$ & Anti-inflammatory & Asante-Kwatia et al. (2020) \\
\hline Anthocleista djalonensis & Loganiaceae & & Antimalaria & Bassey et al. (2009) \\
\hline Antidesma laciniatum & Euphorbiaceae & Leaf & Antimalaria & Titanji et al. (2008) \\
\hline Araliopsis tabuensis & Rutaceae & Stem bark & Antimalaria & Titanji et al. (2008) \\
\hline Artemisia аппиа & Asteraceae & $\mathrm{L}$ & Anti-HIV activity & Noumi and Manga (2011) \\
\hline Asystasia intrusa & Acanthaceae & $\mathrm{L}$ & $\begin{array}{l}\text { Immunomodulatory activity } \\
\text { of methanol extracts }\end{array}$ & Kuate (2015) \\
\hline Azadirachta indica & Meliaceae & $\mathrm{L}, \mathrm{Fl}, \mathrm{Bk}, \mathrm{Se}$ & $\begin{array}{l}\text { Anti-inflammatory, antioxi- } \\
\text { dant, immunomodulatory, } \\
\text { antimalaria }\end{array}$ & $\begin{array}{l}\text { Agbor et al. (2007), Rahmani } \\
\text { et al. (2018), Bashige-Chir- } \\
\text { ibagula et al. (2017) }\end{array}$ \\
\hline Bersama engleriana & Melianthaceae & $\mathrm{L}, \mathrm{Bk}, \mathrm{Rt}$ & $\begin{array}{l}\text { Methanol extracts from } \\
\text { the Laves, bark and roots } \\
\text { inhibited at } 80 \% \text { the activ- } \\
\text { ity of the Human Immuno- } \\
\text { deficiency Virus (HIV) } \\
\text { enzyme }\end{array}$ & Mbaveng et al. (2011) \\
\hline Bersama engleriana & Meliantaceae & Leaf & Antimalaria & Titanji et al. (2008) \\
\hline Bidens pilosa & Asteraceae & Stem bark, & Antimalaria & Titanji et al. (2008) \\
\hline
\end{tabular}


Table 5 (continued)

\begin{tabular}{|c|c|c|c|c|}
\hline Scientific name & Family & Part used & $\begin{array}{l}\text { Existing pharmacological } \\
\text { records }\end{array}$ & References \\
\hline Bobgunia madagascariensis & Fabaceae & & Antimalaria & $\begin{array}{l}\text { Bashige-Chiribagula et al. } \\
\text { (2017) }\end{array}$ \\
\hline Bridelia scleroneura & Euphorbiaceae & $\mathrm{Bk}, \mathrm{Rt}$ & Anti-inflammatory & Kuete and Efferth (2010) \\
\hline Cajanus cajan & Fabaceae & Roots, leaf & Antimalaria & Titanji et al. (2008) \\
\hline Calotropis procera & Apocynaceae & $\mathrm{L}$ & Anti-inflammatory & Asante-Kwatia et al. (2020) \\
\hline Capparis erythrocarpos & Capparidaceae & $\mathrm{L}$ & Anti-inflammatory & Asante-Kwatia et al. (2020) \\
\hline Carica papaya & Caricaceae & $\mathrm{L}, \mathrm{Fr}$ & $\begin{array}{l}\text { Anti-inflammatory, antima- } \\
\text { laria }\end{array}$ & $\begin{array}{l}\text { Sagnia et al. (2014), Sebua } \\
\text { and Maroyi (2013) }\end{array}$ \\
\hline Cassia alata & Caesalpiniaceae & $\mathrm{L}$ & Anti-inflammatory & Sagnia et al. (2014) \\
\hline Cassia occidentalis & Fabaceae & Leaves & Antimalaria & $\begin{array}{l}\text { Bashige-Chiribagula et al. } \\
\text { (2017) }\end{array}$ \\
\hline Cassia sieberiana & Caesalpiniaceae & Rt & Anti-inflammatory & Asante-Kwatia et al. (2020) \\
\hline Caucalis melanantha & Apiaceae & $\mathrm{L}$ & $\begin{array}{l}\text { Immunomodulatory activity } \\
\text { of methanol extracts }\end{array}$ & Kuate (2015) \\
\hline Ceiba pentandra & Bombacaceae & $\mathrm{Bk}$ & Anti-inflammatory & $\begin{array}{l}\text { Agbor et al. (2007), Elion Itou } \\
\text { et al. (2014) }\end{array}$ \\
\hline Clematis chinensis & Ranunculaceae & $\mathrm{L}$ & $\begin{array}{l}\text { Immunomodulatory activity } \\
\text { of methanol extracts }\end{array}$ & Kuate (2015) \\
\hline Cleone rutidosperma & Capparidaceae & Leaf & Antimalaria & Titanji et al. (2008) \\
\hline Combretum molle & Combretaceae & $\mathrm{Bk}$ & Anti-inflammatory & Kuete and Efferth (2010) \\
\hline Commelina diffusa & Commelinaceae & $\mathrm{L}$ & Anti-inflammatory & Asante-Kwatia et al. (2020) \\
\hline Costus afer & Costaceae & St & $\begin{array}{l}\text { Antiviral (chicken pox, influ- } \\
\text { enza, measles and genital } \\
\text { herpes) }\end{array}$ & Ngono Ngane et al. (2011) \\
\hline Cucurbita maxima & Cucurbitaceae & $\mathrm{L}$ & Anti-HIV activity & Noumi and Manga (2011) \\
\hline Cuсurbita реро & Cucurbitaceae & $\mathrm{L}, \mathrm{Rt}$ & $\begin{array}{l}\text { Inhibits HIV-1 reverse tran- } \\
\text { scriptase }\end{array}$ & Noumi and Manga (2011) \\
\hline Cylicodiscus gabinensis & Mimosaceae & $\mathrm{Bk}$ & Anti-oxidant, anti-malarial & $\begin{array}{l}\text { Agbor et al. (2007), Moung- } \\
\text { uengui et al. (2016) }\end{array}$ \\
\hline Cylicodiscus gabunensis & Mimosaceae & Leaf, stem bark & Antimalaria & Titanji et al. (2008) \\
\hline Cymbopogon citratus & Poaceae & $\mathrm{L}$ & Antimalaria & $\begin{array}{l}\text { Etame et al. (2018), Yemele } \\
\text { et al. (2014), Yinyang et al. } \\
\text { (2014) }\end{array}$ \\
\hline Daucus carota & APiaceae & $\mathrm{L}$ & Inhibits HSV-1 replication & Noumi and Manga (2011) \\
\hline Dichaetanthera africana & Melastomataceae & $\mathrm{Bk}$ & Anti-inflammatory & $\begin{array}{l}\text { Oguntibeju (2018), Mokale } \\
\text { Kognou et al. (2017) }\end{array}$ \\
\hline Eleusine indica & Poaceae & $\mathrm{L}$ & Anti-inflammatory & Sagnia et al. (2014) \\
\hline Enantia clorantha & Annonaceae & Stem bark & Antimalaria & Titanji et al. (2008) \\
\hline Entada abyssinica & Mimosaceae & Leaves & Antimalaria & $\begin{array}{l}\text { Bashige-Chiribagula et al. } \\
\text { (2017) }\end{array}$ \\
\hline $\begin{array}{l}\text { Entandrophragma cylindri- } \\
\text { cum }\end{array}$ & Meliaceae & $\mathrm{Bk}$ & Anti-inflammatory & $\begin{array}{l}\text { Fogue Kouam et al. (2012), } \\
\text { Mokale Kognou et al. (2020) }\end{array}$ \\
\hline Enthadrophragma angolense & Meliaceae & Stem bark, leaf & Antimalaria & Titanji et al. (2008) \\
\hline Eremomastax speciosa & Acanthaceae & $\mathrm{L}$ & Anti-inflammatory & Sagnia et al. (2014) \\
\hline Erythrina addisoniae & Caesalpiniaceae & $\mathrm{Bk}$ & Anti-inflammatory & Kuete and Efferth (2010) \\
\hline Erythrina mildbraedii & Caesalpiniaceae & Rt & Anti-inflammatory & Kuete and Efferth (2010) \\
\hline Erythrina sigmoidea & Caesalpiniaceae & Rt & Anti-inflammatory & Kuete and Efferth (2010) \\
\hline Erythrophleum ivorense & Caesalpiniaceae & Not precised & Anti-inflammatory & Asante-Kwatia et al. (2020) \\
\hline ErythrophLum suaveoLns & Caesalpiniaceae & $\mathrm{Bk}$ & Anti-inflammatory & Kuete and Efferth (2010) \\
\hline Euphorbia hirta & Euphorbiaceae & Wp & Antimalaria & Tamo et al. (2016), \\
\hline Ficus exasperata & Moraceae & $\mathrm{L}$ & Anti-inflammatory & Asante-Kwatia et al. (2020) \\
\hline Ficus exasperata & Moraceae & Leaf & Antimalaria & Titanji et al. (2008) \\
\hline
\end{tabular}


Table 5 (continued)

\begin{tabular}{|c|c|c|c|c|}
\hline Scientific name & Family & Part used & $\begin{array}{l}\text { Existing pharmacological } \\
\text { records }\end{array}$ & References \\
\hline Ficus thonningii & Moraceae & Leaf & Antimalaria & Titanji et al. (2008) \\
\hline Glossocalyx brevipes - & Monimiaceae & Leaf & Antimalaria & Titanji et al. (2008) \\
\hline Glyphaea brevis & Tilliaceae & $\mathrm{L}, \mathrm{Bk}$ & Anti-inflammatory & Asante-Kwatia et al. (2020) \\
\hline Gossypium spp. & Malvaceae & Cottonseed & Antimalaria & Titanji et al. (2008) \\
\hline $\begin{array}{l}\text { Harungana madagascarien- } \\
\text { sis }\end{array}$ & Clusiaceae & Bark & ANtimalaria & Weniger et al. (2008) \\
\hline $\begin{array}{l}\text { Harungana madagascarien- } \\
\text { sis }\end{array}$ & Hypericaceae & $\mathrm{Bk}$ & Antimalaria & Ndam et al. (2014) \\
\hline Hexalobus crispiflorus & Annonaceae & Leaf, seed & Antimalaria & Titanji et al. (2008) \\
\hline Hilleria latifolia & Phytolacaceae & $\mathrm{Wp}$ & Anti-inflammatory & Asante-Kwatia et al. (2020) \\
\hline Holarrhena floribunda & Apocynaceae & $\mathrm{Bk}, \mathrm{L}$ & Antimalaria & Yinyang et al. (2014) \\
\hline Jatropha curcas & Euphorbiaceae & Rt & Anti-inflammatory & Asante-Kwatia et al. (2020) \\
\hline Kalanchoe crenata & Crassulacées & Not specified & Anti-inflammatory & Kuete and Efferth (2010) \\
\hline Khaya senegalensis & Meliaceae & $\mathrm{L}, \mathrm{Fr}$ & Antimalaria & $\begin{array}{l}\text { Arbonier (2019), Makut et al. } \\
\text { (2008) }\end{array}$ \\
\hline Lactuca capensis & Asteraceae & Not specified & $\begin{array}{l}\text { Treatment of HIV/AIDS } \\
\text { and related opportunistic } \\
\text { infections }\end{array}$ & Tchuenguem et al. (2017) \\
\hline Landolfia kirkii & Apocynaceae & Leaves, Stem bark & Antimalaria & $\begin{array}{l}\text { Bashige-Chiribagula et al. } \\
\text { (2017) }\end{array}$ \\
\hline Mallotus oppositofolius & Euphorbiaceae & Leaf & Antimalaria & Titanji et al. (2008) \\
\hline Mangifera indica & Anacardiaceae & $\mathrm{Bk}$ & Antimalaria & $\begin{array}{l}\text { Yemele et al. (2014), Yinyang } \\
\text { et al. (2014) }\end{array}$ \\
\hline Milletia griffoniana & Caesalpiniaceae & $\mathrm{L}$ & Anti-inflammatory & Kuete and Efferth (2010) \\
\hline Milletia versicolor & Caesalpiniaceae & $\mathrm{L}$ & Anti-inflammatory & Kuete and Efferth (2010) \\
\hline Millettia griffoniana & $\begin{array}{l}\text { Leguminoceae-Papilio- } \\
\text { noideae }\end{array}$ & Leaf, stem bark & Antimalaria & Titanji et al. (2008) \\
\hline Momordica charantia & Cucurbitaceae & $\mathrm{L}$ & $\begin{array}{l}\text { Immunomodulatory activity, } \\
\text { antiviral (Chicken-pox } \\
\text { Measles Genital herpes } \\
\text { Shingles) }\end{array}$ & $\begin{array}{l}\text { Mahamat et al. (2020), Ngono } \\
\text { Ngane et al. (2011) }\end{array}$ \\
\hline Momordica charantia & Cucurbitaceae & $\mathrm{L}$ & Antimalaria & $\begin{array}{l}\text { Jiofack et al. (2009), Mozaniel } \\
\text { et al. (2018) }\end{array}$ \\
\hline Moringa oleifera & Moringaceae & $\mathrm{L}, \mathrm{Se}$ & $\begin{array}{l}\text { Boost appetite and immunity, } \\
\text { anti-HIV activity }\end{array}$ & Noumi and Manga (2011) \\
\hline Musa paradisiaca & Moraceae & Leaf & Antimalaria & Titanji et al. (2008) \\
\hline Neoboutonia velutina & Euphorbiaceae & Leaf, stem bark & Antimalaria & Titanji et al. (2008) \\
\hline Newbouldia laevis & Bigoniaceae & $\mathrm{L}$ & Anti-inflammatory & Asante-Kwatia et al. (2020) \\
\hline Occimum gratissimum & Lamiaceae & $\mathrm{L}$ & $\begin{array}{l}\text { Immunomodulatory activity } \\
\text { of methanol extracts }\end{array}$ & Kuate (2015) \\
\hline Ocimum basilicum & Lamiaceae & $\mathrm{L}$ & $\begin{array}{l}\text { Inhibits HIV-1 reverse tran- } \\
\text { scriptase }\end{array}$ & Noumi and Manga (2011) \\
\hline Ocimum gratissimum & Lamiaceae & Leaves & & $\begin{array}{l}\text { Bashige-Chiribagula et al. } \\
\text { (2017) }\end{array}$ \\
\hline Odyendyea gabonensis & Simaroubaceae & Leaf, stem bark & Antimalaria & Titanji et al. (2008) \\
\hline Pachypodanthium confine & Annonaceae & Leaf & Antimalaria & Titanji et al. (2008) \\
\hline Palisota hirsuta & Commelinaceae & $\mathrm{L}$ & Anti-inflammatory & Asante-Kwatia et al. (2020) \\
\hline Panda oleosa & Pandaceae & $\mathrm{Bk}$ & $\begin{array}{l}\text { Used in traditional medicine } \\
\text { in Kisangani city to treat } \\
\text { various diseases including } \\
\text { diabetes and HIV/AIDs }\end{array}$ & Muhoya et al. (2017) \\
\hline Peniantus longifolius & Menispermaceae & Stem bark & Antimalaria & Titanji et al. (2008) \\
\hline Pentadiplandra brazzeana & Pentadiplandraceae & Leaf, stem bark & Antimalaria & Titanji et al. (2008) \\
\hline
\end{tabular}


Table 5 (continued)

\begin{tabular}{|c|c|c|c|c|}
\hline Scientific name & Family & Part used & $\begin{array}{l}\text { Existing pharmacological } \\
\text { records }\end{array}$ & References \\
\hline Peperomia vulcanica & Piperaceae & Leaf & Antimalaria & Titanji et al. (2008) \\
\hline Phyllanthus muellerianus & Euphorbiaceae & $\mathrm{Wp}$ & Anti-inflammatory & $\begin{array}{l}\text { Asante-Kwatia et al. (2020), } \\
\text { Ogunwande et al. (2019) }\end{array}$ \\
\hline Phyllantus mиellerianus & Euphorbiaceae & Leaf, stem bark & Antimalaria & Titanji et al. (2008) \\
\hline Picralima nitida & Apocynaceae & $\mathrm{Se}$ & Anti-inflammatory & Asante-Kwatia et al. (2020) \\
\hline Picralima nitida & Apocynaceae & Fr, Rt & Antimalaria & $\begin{array}{l}\text { Tamo et al. (2016), Tsobou } \\
\text { et al. (2015) }\end{array}$ \\
\hline Piper nigrum & Piperaceae & Seed & Antimalaria & Titanji et al. (2008) \\
\hline Piper unbellatum & Piperaceae & Leaf & Antimalaria & Titanji et al. (2008) \\
\hline Polyscias fulva & Araliaceae & $\mathrm{L}$ & Anti-inflammatory & Sagnia et al. (2014) \\
\hline Prunus africana & Rosaceae & $\mathrm{Bk}$ & & Agbor et al. (2007) \\
\hline Pseudocedrela kotschyi & Meliaceae & $\mathrm{Bk}$ & $\begin{array}{l}\text { Extracts stimulate monocyte } \\
\text { proliferation response }\end{array}$ & Oumar et al. (2010) \\
\hline Psidium guayava & Myrtaceae & $\mathrm{L}$ & $\begin{array}{l}\text { Antibacterial, anti-malarial, } \\
\text { anti-diarrhoeal, anti- } \\
\text { inflammatory, antioxidant } \\
\text { activity? antimalaria }\end{array}$ & $\begin{array}{l}\text { Agbor et al. (2007), Titanji } \\
\text { et al. (2008), Kaur et al. } \\
\text { (2018) }\end{array}$ \\
\hline Pteleopsis hylodendrom & Combretaceae & $\mathrm{Bk}$ & $\begin{array}{l}\text { Antiviral (chicken pox, influ- } \\
\text { enza, measles and genital } \\
\text { herpes) }\end{array}$ & Ngono Ngane et al. (2011) \\
\hline Pycnanthus angolensis & Myrtaceae & Leaf, stem bark & Antimalaria & Titanji et al. (2008) \\
\hline Raphanus sativus & Brassicaceae & $\mathrm{L}, \mathrm{Bk}, \mathrm{Rt}$ & Antiviral activity & Noumi and Manga (2011) \\
\hline Rauvolfia macrophylla & Apocynaceae & Stem bark & Antimalaria & Weniger et al. (2008) \\
\hline Rauvolfia vomitoria & Apocynaceae & Stem bark & Antimalaria & Titanji et al. (2008) \\
\hline Reneilmia cincinnata & Zingiberaceae & Fruit & Antimalaria & Titanji et al. (2008) \\
\hline $\begin{array}{l}\text { Schumanniophyton magnifi- } \\
\text { cum }\end{array}$ & Rubiaceae & Stem bark & Antimalaria & Titanji et al. (2008) \\
\hline Sclerocarya birrea & Anacardiaceae & $\mathrm{Bk}$ & Anti-inflammatory & Kuete and Efferth (2010) \\
\hline Scoparia dulcis & Scrophulariaceae & Whole plant & Antimalaria & Titanji et al. (2008) \\
\hline Senna alata & Caesalpiniaceae & $\mathrm{L}$ & $\begin{array}{l}\text { Inhibits HIV-1 reverse tran- } \\
\text { scriptase }\end{array}$ & Noumi and Manga (2011) \\
\hline Solanum torvum & Solanaceae & $\mathrm{L}$ & Anti-inflammatory & Kuete and Efferth (2010) \\
\hline Spathodea campanulata & Bignoniaceae & $\mathrm{L}, \mathrm{Bk}$ & $\begin{array}{l}\text { Anti-inflammatory, antioxi- } \\
\text { dant } \\
\text { Antiviral (Chicken-pox } \\
\text { Genital herpes) }\end{array}$ & $\begin{array}{l}\text { Pone Kamdem (2017), Etame } \\
\text { et al. (2018), Ngono Ngane } \\
\text { et al. (2011) }\end{array}$ \\
\hline Stachytapheta cayenensis & Verbenaceae & Leaf & Antimalaria & Titanji et al. (2008) \\
\hline $\begin{array}{l}\text { Stereospermum acuminatis- } \\
\text { simum }\end{array}$ & Bignoniaceae & Bark & Antimalaria & Weniger et al. (2008) \\
\hline Stereospermum zenkeri & Bignoniaceae & Bark & Antimalaria & Weniger et al. (2008) \\
\hline Strychnos icaja & Loganiaceae (?) & Root & Antimalaria & Titanji et al. (2008) \\
\hline Symphonia globulifera & Clusiaceae & Bark & Antimalaria & Weniger et al. (2008) \\
\hline Synedrella nodiflora & & $\mathrm{Wp}$ & Anti-inflammatory & Asante-Kwatia et al. (2020) \\
\hline $\begin{array}{l}\text { Tapinanthus globiferus } \\
\text { (harvested from Persea } \\
\text { americana) }\end{array}$ & Loranthaceae & $\mathrm{L}$ & $\begin{array}{l}\text { Anti-inflammatory, immu- } \\
\text { nomodulatory and antioxi- } \\
\text { dant properties }\end{array}$ & Gounoue et al. (2019) \\
\hline Thomandersia hensii & Acanthaceae & Leaves, stem bark & Antimalaria & Titanji et al. (2008) \\
\hline Trichilia emetica & Meliaceae & Bark & Antimalaria & Diarra et al. (2015) \\
\hline Trichilia monadelpha & Meliaceae & $\mathrm{Bk}$ & Anti-inflammatory & Asante-Kwatia et al. (2020) \\
\hline Turreanthus africanus & Meliaceae & seed & Antimalaria & Titanji et al. (2008) \\
\hline Uapaca guineensis & Euphorbiaceae & Not specified & Anti-inflammatory & Kuete and Efferth (2010) \\
\hline Vernonia amygdalina & Asteraceae & $\mathrm{L}$ & Anti-inflammatory & Asante-Kwatia et al. (2020) \\
\hline
\end{tabular}


Table 5 (continued)

\begin{tabular}{|c|c|c|c|c|}
\hline Scientific name & Family & Part used & $\begin{array}{l}\text { Existing pharmacological } \\
\text { records }\end{array}$ & References \\
\hline Vernonia amygdalina & Asteraceae & $\mathrm{L}, \mathrm{Rt}$ & Anti-HIV activity & Noumi and Manga (2011) \\
\hline Vitellaria paradoxa & Sapotaceae & $\mathrm{Bk}$ & Anti-inflammatory & Foyet et al. (2015) \\
\hline Vitex thyrsiflora & & $\mathrm{Bk}$ & Anti-inflammatory & Mokale Kognou et al. (2020) \\
\hline Voacanga africana & Apocynaceae & $\mathrm{Bk}$ & Antioxidant & $\begin{array}{l}\text { Adu et al. (2015), Agbor et al. } \\
\text { (2007) }\end{array}$ \\
\hline Xylopia aethiopica & Annonaceae & Fr & Anti-inflammatory & Asante-Kwatia et al. (2020) \\
\hline Xylopia parviflora, & Annonaceae & Seed & Antimalaria & Titanji et al. (2008) \\
\hline Xylopia phloiodora, & Annonaceae & Seed & Antimalaria & Titanji et al. (2008) \\
\hline Xymolox monosperma & Annonaceae & Leaf, stem bark & Antimalaria & Titanji et al. (2008) \\
\hline Zanthoxylum heitzii & Rutaceae & $\mathrm{Bk}$ & $\begin{array}{l}\text { Antioxidant, antimalarial, } \\
\text { antiinfmalatory, immu- } \\
\text { norestorative }\end{array}$ & $\begin{array}{l}\text { Agbor et al. (2007), Sadeer } \\
\text { et al. (2019), Mokondjimobe } \\
\text { et al. (2012) }\end{array}$ \\
\hline Zingiber officinale & Zingiberaceae & $\mathrm{L}, \mathrm{Rt}$ & $\begin{array}{l}\text { Antimalaria, anti-HIV-1 } \\
\text { activity }\end{array}$ & $\begin{array}{l}\text { Titanji et al. (2008), Noumi } \\
\text { and Manga (2011) }\end{array}$ \\
\hline Ziziphus abyssinica & Rhamnaceae & $\mathrm{Rt}$ & Anti-inflammatory & Asante-Kwatia et al. (2020) \\
\hline
\end{tabular}

${ }^{*} \mathrm{~L}=$ leave, $\mathrm{Bk}=$ bark, $\mathrm{St}=\mathrm{Stem}, \mathrm{Rt}=$ roots, $\mathrm{Fr}=$ fruit, $\mathrm{Se}=$ seed, $\mathrm{Fl}=$ flower, Tbk $=$ root bark, $\mathrm{Wp}=$ whole $\mathrm{plant}, \mathrm{Lx}=$ latex

Fig. 3 Frequency distribution of plant parts with imunomodulatory, anti-inflammatory or antiviral properties

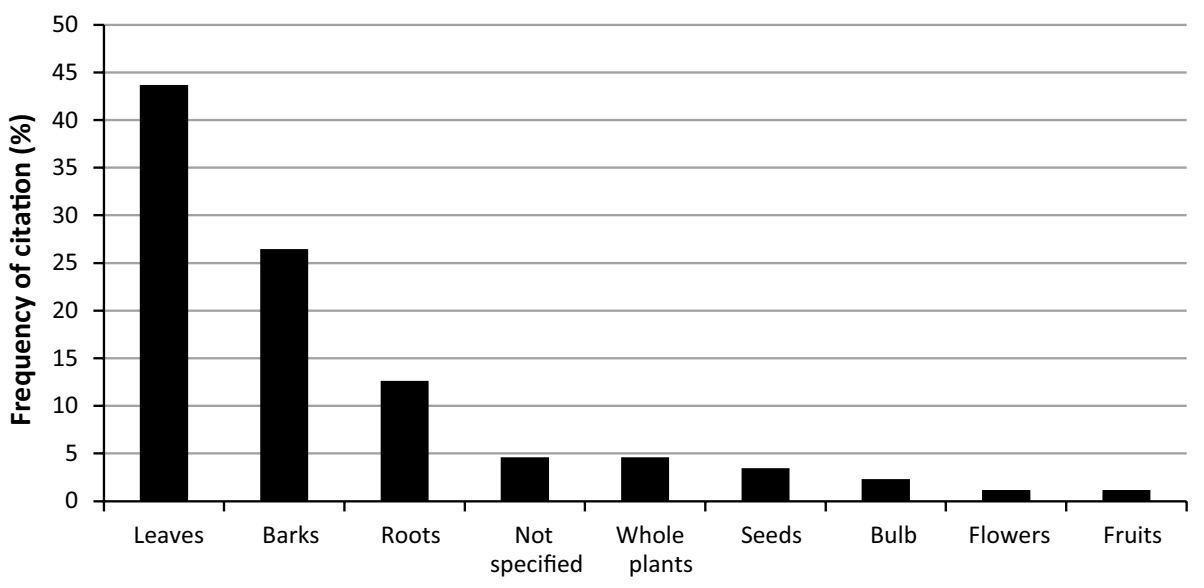

\section{Conservation of medicinal plants and documentation of available knowledge on their use}

In the face of the current risk of deforestation and degradation, conservation of medicinal plants must be a central focus. In this regard, one of the challenges is the lack of a complete and conserved knowledge repository on the national pharmacopoeia and the immense medicinal metabolite diversity among these plants. Such a repository will be vital in providing the scientific community with comprehensive knowledge about metabolite diversity and exploitation in early response strategies for emerging diseases. Because of the growing environmental degradation and the rapid loss of the natural habitat for some of these plants due to anthropogenic activities, it is becoming increasingly urgent to reinforce medicinal plants conservation and documentation of their uses.

To ensure the sustainability of the resource base and to address potential risk of overexploitation that may result from excessive commercialization and unsustainable practices, conservation measures for medicinal plants will also be required. The effectiveness of the future sustainability of local natural ecosystems that harbour these medicinal plants will depend upon conservation management approaches that value the importance of involving local communities. In this light, there are lessons learned from Prunus africana management in the Mount Cameroon area that can fuel our steps forward in the establishment of such a medicinal plant conservation strategy.

The ratification by Cameroon of the Nagoya protocol on access to genetic resources and benefit sharing opens new and promising avenues to achieve the objectives of 


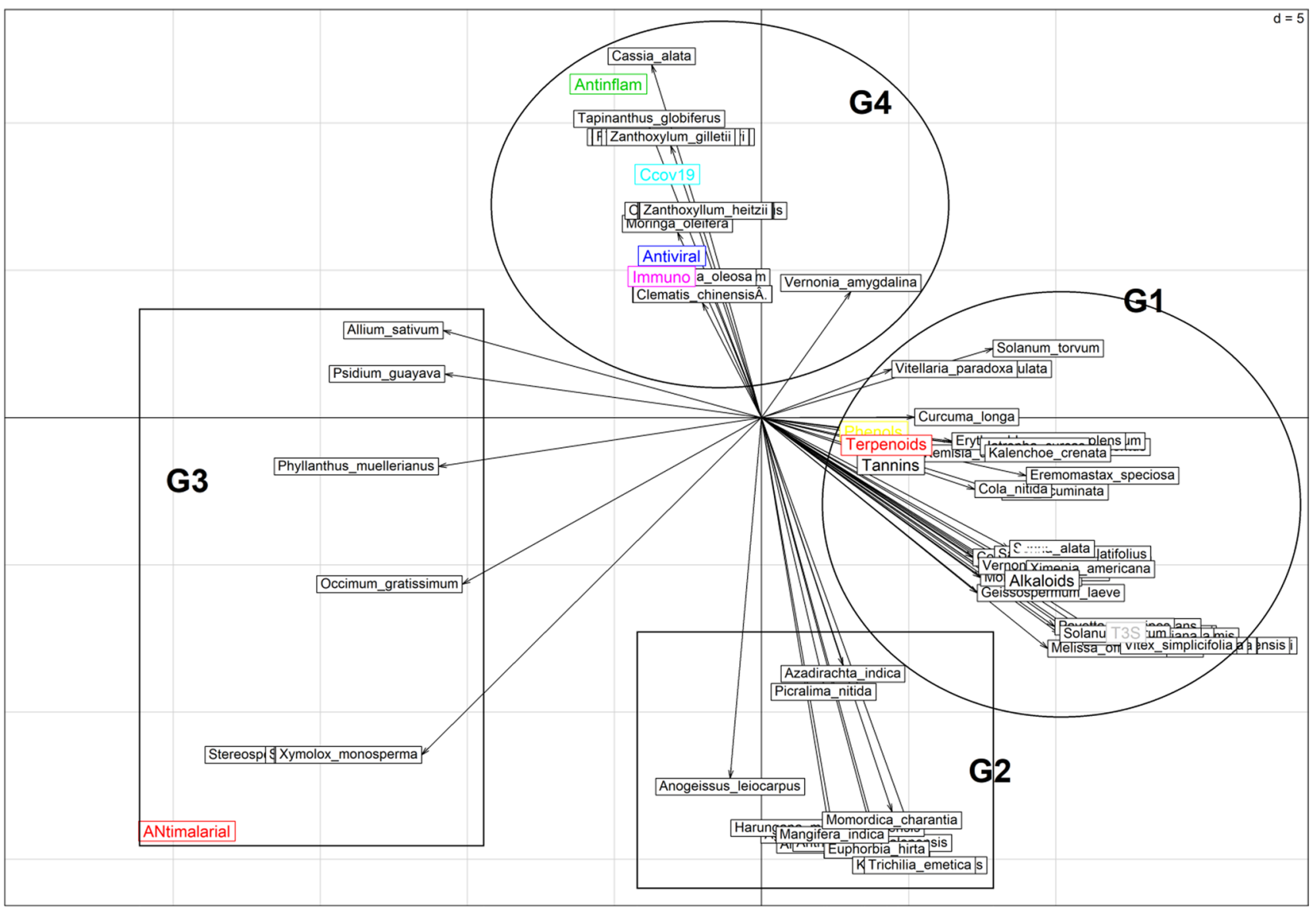

Fig. 4 Results of PCA ordination showing patterns of variability among the 230 species recorded

conserving local medicinal plants, ensuring their sustainable utilization and improving their contribution in livelihoods improvement and economic development.

\section{Encouraging private sector involvement in herbal drug development}

There has so far been only very poor participation of the local private pharmaceutical industries in the field of herbal drug development in Cameroon. There should be incentives developed to attract and stimulate their investment in traditional medicine research, development and commercialization.

\section{Conclusion}

The purpose of this stock-taking study was to provide a preliminary review on Cameroonian medicinal plants with the potential to be evaluated and developed as remedies for the management of COVID-19. It appears that the country's medicinal plants potential is immense and a promising resource from a perspective of novel drug development against this pandemic. Based on the present findings it can be concluded that medicinal plants can be promising resources for the management of COVID-19 in African herbal medicine in general and Cameroon in particular.

Despite the great potential of local medicinal plants, it is unfortunate that they are still pejoratively refered to as "grand-mother recipes". More than ever, there is a need for applied research to provide more scientific evidence for the efficacy, to establish the standard formulation using the preliminary check list presented in this review and further clinical studies as part of the response strategy for the management of COVID-19.

Acknowledgements The authors are grateful to Carol J. Pierce Colfer (Center for International Forestry Research in Bogor, Indonesia and Cornell University in Ithaca, New York, United States of America) for the valuable comments during the writing of the paper and for proofreading the final manuscript.

Funding The authors declare that they did not receive any funding for carrying out this research. 


\section{Declarations}

Ethical statement This article does not contain any studies with human participants or animals performed by any of the authors.

Conflict of interest Fongnzossie Fedoung Evariste has no conflict of interest. Biwole Achille Bernard has no conflict of interest. Nyangono Biyegue Christine Fernande has no conflict of interest. Ngansop Tounkam Marlène has no conflict of interest. Patrick Akono Ntonga has no conflict of interest. Nguiamba Veronique Priscille has no conflict of interest. Essono Damien Marie has no conflict of interest. Forbi Preasious Funwi has no conflict of interest. Tonga Calvin has no conflict of interest. Nguenang Guy Merlin has no conflict of interest. Kemeuze Victor has no conflict of interest. Sonwa Denis Jean has no conflict of interest. Tsabang Nole has no conflict of interest. Isabelle Sandrine Bouelet has no conflict of interest. Tize Zra has no conflict of interest. Boum Alexandre Teplaira has no conflict of interest. Momo Solefack Marie Caroline has no conflict of interest. Betti Jean Lagarde has no conflict of interest. Nouga Bissoue Achille has no conflict of interest. Lehman Leopold Gustave has no conflict of interest. Mapongmetsem Pierre Marie has no conflict of interest. Nneme Nneme Leandre has no conflict of interest. Ngono Ngane Annie has no conflict of interest. Ngogang Yonkeu Jeanne has no conflict of interest.

\section{References}

Abonyi DO, Adikwu MU, Esimone CO, Ibezim EC (2009) Plants as sources of antiviral agents. Afr J Biotechnol 8(17):3989-3994

Adem S, Eyupoglu V, Sarfraz I, Rasul A, Ali M. (2020) Identification of Potent COVID-19 Main Protease (Mpro) Inhibitors from Natural Polyphenols: An in Silico Strategy Unveils a Hope against Corona. Preprints. DOI: https://doi.org/10.20944/preprints2 02003.0333.v1. Available from: https://www.researchgate.net/ publication/340095736_Identification_of_Potent_COVID19_ Main_Protease_Mpro_Inhibitors_from_Natural_Polyphenols_ An_in_Silico_Strategy_Unveils_a_Hope_against_CORONA [accessed Apr 21 2020].

Adu F, Apenteng JA, Akanwariwiak WG, Sam GH, Ntinagyei Mintah D, Bortsie EB (2015) Antioxidant and in-vitro anthelminthic potentials of methanol extracts of barks and leaves of Voacanga africana and Rauwolfia vomitoria. Afr J Microbiol Res 9(35):1984-1988

Agbedahunsi JM, Elujoba AA, Makinde JM, Oduda AMJ (1998) Antimalarial Activity of Khaya grandifoliola Stem-bark. Pharm Biol 36(1):8-12

Agbor GA, Kuate D, Oben JE (2007) Medicinal plants can be good source of antioxidants: Case study in Cameroon. Pak J Biol Sci 10(4):537-550

Ahmad HA (2014) Review on Anogeissus leiocarpus, a potent african traditional drug. Int J Res Pharm Chem 4(3):496-500

Akram M, Ahmed A, Shahab-Uddin K, Usmanghani K, Hannan A, Mohiuddin E, Asif M (2010) Curcuma longa and curcumin: a review article. Roman J Biol Plant Biol 55(2):65-70

Alamgeer Younis W, Asif H, Sharif A, Riaz H, Bukhari IA, Sssiri AM (2018) Traditional medicinal plants used for respiratory disorders in Pakistan: a review of the ethno-medicinal and pharmacological evidence. Chin Med 13:48

Alowanou GG, Olounlade AP, Azando EV, Dedehou VFGN, Daga FD, Hounzangbeadote SM (2015) A review of Bridelia ferruginea, Combretum glutinosum and Mitragina inermis plants used in zootherapeutic remedies in West Africa: historical origins, current uses and implications for conservation. J Appl Biosci 87:8003-8014
Amoa Onguéné P, Ntie-Kang T, Likowo Lifongo L, Ndom N, Sippl W, Meva'a Mbaze L (2013) The potential of anti-malarial compounds derived from African medicinal plants, part I: a pharmacological evaluation of alkaloids and terpenoids. Malar J 12:449

Anywar G, Kakudidi E, Byamukama R, Mukonzo J, Schubert A, Oryem-Origa H (2020) Data on medicinal plants used by herbalists for boosting immunity in people living with HIV/ AIDS in Uganda. Data Brief 29(2020): 105097

Arbonnier M (2019) Arbres, arbustes et lianes d'Afrique de l'Ouest. Quatrième édition, Éditions Quae-Cirad, p 2019

Asante-Kwatia E, Yeboah Mensah A, Frimpong Baidoo M (2020) Analgesic and anti-inflammatory effect of ghanaian medicinal plants. In: Intec open Eds. Medicinal plants - use in prevention and treatment of diseases

Asongalem EA, Foyet HS, Ekobo S, Dimo T, Kamtchouing P (2004) Antiinflammatory, lack of central analgesia and antipyretic properties of Acanthus montanus (Ness) T. Anderson. J Ethnopharmacol. 95(1):63-8

Azantsa Kingue GB, Djuikoo Nouteza I, Kuikoua Tchetmi W, Takuissu G, Ngondi JL, Oben J (2017) Phytochemical Screening and Antidiabetic evaluation of Citrus sinensis Stem Bark Extracts. Int J Biochem Res \& Rev 17(2):1-13

Bogninou GSR, Bigo Agadaba PH, Gnanwe M, Agbangnan DCP, Chabi NW, Yedomonhan H, Avlessi F (2018) Phytochemical composition and antioxydant capacity of abelmoschus esculentus 1. Fresh Immature Fruits. Am J Food Sci Technol 6(5):223-227

Calina D, Sarkar C, Arsene AL, Salehi B, Docea AO, Mondal M, Islam MT, Zali A, Sharifi-Rada J (2020) Recent advances, approaches and challenges in targeting pathways for potential COVID-19 vaccines development. Immunol Res 68:315-324

Chah KF, Muko KN, Oboegbulm SI (2000) Antimicrobial activity of methanolic extract of Solanum torvum fruit. Fitoterapia 71(2):187-189

Chinsembu KC, Hedimbi M (2010) An ethnobotanical survey of plants used to manage HIV/AIDS opportunistic infections in Katima Mulilo, Caprivi region, Namibia. J Ethnobiol Ethnom 6:25

Colson P, Rolain JM, Lagier JC, Brouqui P, Raoult D (2020) Chloroquine and hydroxychloroquine as availabL weapons to fight COVID-19. Int J Antimicrob Ag 4:105932

Crtv 2020. COVID-19: Archbishop Samuel Kleda proposes a herbal remedy. Retreived on http://www.crtv.cm/2020/04/COVID-19archbishop-samuel-kleda-proposes-a-herbal-remedy/, 29 April 2020.

Cunningham AB, Shanley P, Laird S (2008) Health, habitats and medicinal plant use. In: Pierce Colfer CJ (ed) Human health and forests: a global overview of issues, practice and policy. Eathscan, London

Curbelo J, Luquero Bueno S, Galva Roman JM, Ortega-Gomez M, Rajas O, Fernandez-Jimenez G, Vega-Pirisi L, RodriguezSalvanes F, Arnalich B (2017) Díaz A, Costa R, de la Fuente H, Lancho A, Suarez C, Ancochea J, Aspa J (2017) Inflammation biomarkers in blood as mortality predictors in communityacquired pneumonia admitted patients: Importance of comparison with neutrophil count percentage or neutrophil-lymphocyte ratio. PLoS ONE 12(3):e0173947

Damrongkiet A, Svasti J, Kittakoop P, Khachonwut DP, Tanticharoen M, Thebtaranonth Y (2002) Antiviral isoflavonoid sulfate and steroidal glycosides from the fruits of Solanum torvum. Phytochemistry 59(4):459-463

Dibong DS, Mvogo OBP, Vandi D, Ndjib RC, Tchamaha MF, Mpondo ME (2015) Ethnobotanique des plantes médicinaLs anti hémorroïdaires des marchés et villages du Centre et du Littoral Cameroun. J Appl Biosci 96:9072-9093

ECDC 2020. COVID-19 (2020) Situation update worldwide, as of 10 august 2020. Retreived from https://www.ecdc.europa.eu/en/ geographical-distribution-2019-ncov-cases, on January 13, 2021. 
Elion Itou RDG, Sanogo R, Etou Ossibi AW, Nsondé Ntandou FG, Ondelé R, Pénem BM, Okiémy Andissa N, Diallo D, Ouamba JM, Abena AA (2014) Anti-inflammatory and analgesic effects of aqueous extract of stem bark of ceiba pentandra gaertn. Pharmacol Pharmacy 5:1113-1118

Etame G, Ngoul CC, Mbome B, Kidik PC, Ngene JP, Yinyang J, Ebongue OC, Ngaba GP, Dibong DS (2018) Contribution à l'étude des plantes médicinaLs et Lurs utilisations traditionnelLs dans L département du Lom et Djerem (Est, Cameroun). J Anim Pl Sci 35(1):5560-5578

Faheem Khan M, Khan MA, Khan ZA, Ahamad T, Ansari WA (2020) Identification of dietary molecules as therapeutic agents to combat COVID-19 using molecular docking studies. Res Square Preprint 2020:20p

Fogue Kouam S, Kusari S, Lamshöft M, KamgueTatuedom O, Spiteller M, Sapelnins G-J (2012) Phytochemistry 83:79-86

Fongnzossie EF, Tize Z, Fogang Nde PJ, Nyangono Biyegue CF, Bouelet Ntsama IS, Dibong SD, Nkongmeneck BA (2017) Ethnobotany and pharmacognostic perspective of plant species used as traditional cosmetics and cosmeceuticals among the Gbaya ethnic group in Eastern Cameroon. S Afr J Bot 112:29-39

Fongod AGN, Modjenpa NB, Veranso MC (2013) Ethnobotany of Acanthaceae in the Mount Cameroon region. J Med Pl Res 7(38):2859-2866

Foyet HS, Tsala DE, Zogo Essono Bodo JC, Azanfack Name C, Toussoumna HL, Oben Eyong K (2015) Anti-inflammatory and antiarthritic activity of a methanol extract from Vitellaria paradoxa stem bark. Pharmacog Res 7(74):367-377

Gao J, Tian Z, Yang X (2020) Breakthrough: chloroquine phosphate has shown apparent efficacy in treatment of COVID-19 associated pneumonia in clinical studies. BioSci Trends 14(1):72-73

Gardini F, Belletti N, Ndagijimana M, Guerzoni ME, Tchoumbougnang F, Amvam Zollo PH, Claudio Micci C, Rosalba Lanciotti R, Kamdem SLS (2009) Composition of four essential oils obtained from plants from Cameroon, and their bactericidal and bacteriostatic activity against Listeria monocytogenes, Salmonella enteritidis and Staphylococcus aureus. Afr J Microbiol Res 3(5):264-271

Goothy SSK, Goothy S, Choudhary A, Potey GG, Chakraborty H, Kumar AHS (2020) Mahadik VK (2020) Ayurveda's Holistic LifestyL Approach for the Management of Corona virus disease (COVID-19): Possible Role of Tulsi. Int $\mathrm{j}$ Res Pharm Sci 1:16-18

Gounoue KR, Noukeu KBA, Tsakem NMJ, Youmsi FD, Ngueguim TF, Dzeufiet DPD, Dimo T (2019) Anti-inflammatory potential of Tapinanthus globiferus (Loranthaceae) leaves as an alternative treatment against arthritis. J Phytopharmacol 8(3):96-103

Hong-Zhi Du, Xiao-Ying H, Yu-Huan M, Bi-Sheng H (2020) Traditional Chinese Medicine: an effective treatment for 2019 novel coronavirus pneumonia (NCP) $[\mathrm{J}]$. Chin J Nat Med 18(3):206-210

Hossain MF, Akhtar S, Anwar M (2015) Nutritional value and medicinal benefits of pineapple. Int J Nut Food Sci 4(1):84-88

Huang C, Wang Y, Li X, Ren L, Zhao J, Hu Y et al (2020) Clinical features of patients infected with 2019 novel coronavirus in Wuhan. China Lancet. https://doi.org/10.1016/S0140-6736(20)30183-5

Ikpa CBC, Maduka TOD, Ebere Enyoh C, Ikezu Uju JM (2020) Potential plants for treatment and management of COVID-19 in Nigeria. Academic Journal of Chemistry 5(6):69-80

Iqbal S, Younas U, Chan KW, Zia-Ul-Haq M, Ismail M (2012) Chemical composition of Artemisia annua L. leaves and antioxidant potential of extracts as a function of extraction solvents. Molecules 17:6020-6032

Isemura M (2019) Catechin in human health and disease. Molecules 24:528. https://doi.org/10.3390/molecules24030528

Ismail AB, Mohamed EA, Marghany MR, Abdel-Motaal FF, Abdel-Farid IB (2014) d El-Sayed, MA (2014) Preliminary phytochemical screening, plant growth inhibition and antimicrobial activity studies of Faidherbia albida legume extracts. Journal of the Saudi Society of Agricultural Sciences 15:112-117

Jassim SA, Naji MA (2003) Novel antiviral agents: a medicinal plant perspective. J Appl Microbiol 95(3):412-427

Jiofack T, Fokunang C, Kemeuze V, Fongnzossie E, Tsabang N, Nkuinkeu R, Mapongmetsem PM (2008) Ethnobotany and phytopharmacopoea of the SouthWest ethnoecological region of Cameroon. J Med Pl Res 2(8):197-207

Jiofack TR, Ayissi I, Fokunang C, Guedje N, Kemeuze V (2009) Kemeuze V (2009) Ethnobotany and phytomedicine of the upper Nyong valley forest in Cameroon. Afr J Pharm Pharmacol 3(4): $144-150$

Jossang A, Pousset JL, Bodo B (1994) Combreglutinin, a hydrolyzable tannin from Combretum glutinosum. J Nat Prod 55(6):732-737

Kamatou GPP, Vermaak I (2011) Viljoen AM (2011) An updated review of Adansonia digitata: a commercially important African tree. S Afr J Bot 77:908-919

Kamtchueng MO, Balyan R, Mouokeu RS, Tume C, Banerjee C, Chawla SA, Oumar M, Kuiate JR (2017) Anti-inflammatory activity of methanol extract and fractions from Alchemilla kiwuensis Engl. on LPS activated macrophages. Int J Pharmacog Phytochem Res 9(4):473-481

Kaur M, Singh J, Mirza A (2018) Pharmacological and medicinal properties of psidium guajava: a review. Res J Chem Environ Sci 6(4):70-73

Keyaerts E, Vijgen L, Pannecouque C, Van Damme E, Peumans W, Egberink H, Balzarini J, Van Ranst M (2007) Plant Lctins are potent inhibitors of coronaviruses by interfering with two targets in the viral replication cycle. Antivir Res 75:179-187

Khaerunnisa S, Kurniawan H, Awaluddin R, Suhartati S, Soetjipto S (2020) Potential inhibitor of COVID-19 main protease (Mpro) from several medicinal plant compounds by molecular docking study. Preprints 2020:2020030226

Khalifa I, Zhu W, Nafie MS, Dutta K, Li C (2020) Anti-COVID-19 effects of ten structurally different hydrolysable tannins through binding with the catalytic-closed sites of COVID-19 main protease: An in-silico approach. AvailabL from: https://www.resea rchgate.net/publication/339999895_Anti-COVID-19_effects_of_ ten_structurally_different_hydrolysabL_tannins_through_bindi ng_1_with_the_catalytic-closed_sites_of_COVID-19_main protease_An_in-silico_approach_2 [accessed Apr 21 2020]. 2020.

Khodadadi S (2015) Role of herbal medicine in boosting immune system. Immunopathol Persa 1(1):e01

Klimek-Szczykutowicz M, Szopa A, Ekiert H (2020) Citrus limon (Lmon) phenomenon-a review of the chemistry, pharmacological properties, applications in the modern pharmaceutical, food, and cosmetics industries, and biotechnological studies. Plants 9:1-24

Kuate JR (2015) Immunomodulatory activity of methanol Laf extracts of Cameroonian medicinal plants. J Compl Integr Med 12(4):267-275

Kuete V, Efferth T (2010) Cameroonian medicinal plants: pharmacology and derived natural products. Front Pharmacol. https://doi. org/10.3389/fphar.2010.00126

Kuete V, Sipowo VR, Mbaveng AT, da Silva VC, Rodrigues CM, Nkengfack AE, dos Santos LC, Vilgas W (2018) Efferth T (2018) Catechin Derivatives from Parkia biglobosa Displayed SeLctive Cytotoxicity Towards Lukemia CCRF-CEM Cell Line and its PGlycoprotein Expressing Subline CEM/ADR5000. Invest Med Chem Pharmacol. 1(1):1-5

Lai CC, Shih TP, Ko WC, Tang HJ (2020) Hsueh PR (2020) Severe acute respiratory syndrome coronavirus 2 (SARS-CoV-2) and coronavirus disease-2019 (COVID-19): The epidemic and the challenges. Int J Antimicrob Ag 2020(5):105924 
Lelesius R, Karpovaitė A, Mickienè R, Drevinskas T, Tiso N, Ragažinskienè O, Kubilienè L, Maruška A (2019) Šalomskas A (2019) In vitro antiviral activity of fifteen plant extracts against avian infectious bronchitis virus. BMC Veter Res 15:178

Le Point International (2020) Au Mexique, des amulettes contre le coronavirus. Retreived March 23, 2020, from https://www.lepoi nt.fr/monde/au-mexique-des-amulettes-contre-lecoronaviru-2303-2020-2368294_24.php

Liu C, Zhou Q, Li Y, Garner LV, Watkins SP, Carter LJ, Smoot J, Greg AC, Daniels AD, Jervey S, Albaiu D (2020) Research and development on therapeutic agents and vaccines for COVID-19 and related human coronavirus diseases. ACS Cent Sci. 6:315-331

Lu H (2020) Drug treatment options for the 2019-new coronavirus (2019-nCoV). Biosci Trends. https://doi.org/10.5582/bst.01020

Mahomoodally MF (2013) Traditional medicines in Africa: An appraisal of ten potent African medicinal plants. Evidence-based complementaryand alternative medicine. Special Issue on Recent Advances towards Validating Efficacy and Safety of African Traditional Medicines. https://doi.org/10.1155/2013/617459.

Mambe FT, Voukeng IK, Beng VP (2016) Kuete V (2016) Antibacterial activities of methanol extracts from Alchornea cordifolia and four other Cameroonian plants against MDR phenotypes. $\mathbf{J}$ Taibah Univ Med Sci 11(2):121-127

Mba Nguekeu YM, Awouafack MD, Tane P, Nguedia Lando MR, Kodama T, Morita H (2017) A kaempferol triglycoside from Tephrosia preussii Taub (Fabaceae). Nat Prod Res 31(21):2520-2526

Mbaveng TA, Kuete V, Mapunya MB, Beng PV, Nkengfack EA, Meyer MJJ (2011) Lall, N (2011) Evaluation of four Cameroonian medicinal plants for anticancer, antigonorrheal and antireverse transcriptase activities. Environ Toxicol Pharmacol 32(2):162-167

Midi-Madagascar (2020). «COVID-Organics » : La communauté scientifique sort du silence. Retrieved from http://www.midi-madag asikara.mg/COVID-19/2020/04/22/COVID-organics-la-commu naute-scientifique-sort-du-silence/, 22 april 2020.

Ming LC (1999) Ageratum conyzoides: A tropical source of medicinal and agricultural products. In: Janick J (ed) Perspectives on new crops and new uses. ASHS Press, Alexandria, VA, pp 469-473

Moghadamtousi SZ, Kadir HA, Hassandarvish P, Tajik H, Abubakar S, Zandi K (2014) A Review on Antibacterial, Antiviral, and Antifungal Activity of Curcumin. BioMed Research International Volume 2014, Special issue on Biologic Activity and Biotechnological Development of Natural Products. Hindawi Publishing Corporation

Moghadamtousi SZ, Fadaeinasab M, Nikzad S, Mohan G, Ali H, Kadir H (2015) Annona muricata (Annonaceae): a review of its traditional uses, isolated acetogenins and biological activities. Int $\mathbf{J}$ Mol Sci 16(7):15625-15658

Mohal DS, Dewani AP, Chandewar AV, Khadse CD, Tripathi AS (2009) Agrawa SS (2009) Brief review on medicinal potential of Terminalia catappa. J Herb Herbal Med Toxicol 3(1):13-17

Mohamad M, Ali MW, Ahmed A (2010) Modelling for extraction of major components from Eurycoma longifolia. J Appl Sci 10(21):2572-2577

Mohammadi N, Shaghaghi N (2020) Inhibitory Effect of Eight Secondary Metabolites from Conventional Medicinal Plants on COVID_19 Virus Protease by MoLcular Docking Analysis. ChemRxiv. Preprint. https://doi.org/10.26434/chemrxiv.11987 475.v1

Mokale Kognou AL, Ngono Ngane RA, Pawar RS, Pateriya P, Pal PN, Ebelle Etame RM, Tiabou Tchinda A, Agbor Agbor G, Kumar S, Mouokeu RS, Sone Mouelle A (2017) Enguehard Gueiffier S (2017) Anti-Inflammatory Activity and Bioavailability Profile of Ethanolic Extract of Dichaetanthera africana. Pharmacologia $8(1): 32-40$
Mokale Kognou AL, Kowa TK, Pateriya P, Pal NP, Mouokeu RS, Tchinda Tiabou A, Agbor Agbor G, Singh RP (2020) Ngono Ngane RA (2020) Pharmacological evidence of Vitex thyrsiflora, Entandrophragma cylindricum, and Anonidium mannii used forthe management of inflammation in Cameroon. J Basic Clin Physiol Pharmacol 2020:20190053

Mokondjimobe E, Joe MB, Barkha S, Dzeufiet PD, Chenal H, Otsudi' andjeka JB, Bipolo S, Besse M, Mamadou G, Nzouzi NL, Kamtchouing P, Meddah B, Okpwae J, Schobiltgen F (2012) Eto B (2012) Fagaricine, a new immunorestorative phytomedicine from Zanthoxylum heitzii: Preclinical and multicenter cohort clinical studies based on HIV-infected patients in six countries. Phytopharmacology 2(1):26-45

Moradi MT, Karimi A, Lorigooini Z (2017) Alkaloids as the natural anti-influenza virus agents: a systematic review. Toxin Rev. https://doi.org/10.1080/15569543.2017.1323338

Mounguengui S, Saha Tchinda JB, Ndikontar MK, Dumarçay S, Attéké C, Perrin D, Gelhaye G (2016) Gérardin P (2016) Total phenolic and lignin contents, phytochemical screening, antioxidant and fungal inhibition properties of the heartwood extractives of ten Congo Basin tree species. Ann For Sci 73(2):287-296

Muhoya FK, Kadima JN, Ranarivelo N, Frédérich M, Hubert P, Djang'eing'a RM (2017) Preliminary phytochemical content and antidiabetic potential investigations of Panda oleosa (Pierre) used in Kisangani Areas. Am J Anal Chem. 8:564-581

Mutalik S, Paridhavi K, Mallikarjuna Rao C (2003) Udupa N (2003) Antipyretic and analgesic effect of leaves of Solanum melongena Linn rodents. Ind J of Pharmacol 35:312-315

Ndam LM, Mih AM, Fongod AGN, Tening AS, Tonjock RK, Enang JE (2014) Fujii Y (2014) Phytochemical screening of the bioactive compounds in twenty (20) Cameroonian medicinal plants. Int J Curr Microbiol Appl Sci 3(12):1-11

Ndjonka D, Bergmann B, Agyare C, Zimbres FM, Lüersen K, Hensel A, Wrenger C, Liebau E (2008) In vitro activity of extracts and isolated polyphenols from West African medicinal plants against Plasmodium falciparum. Parasit Res 111(2):827-834

Ngaha NMI, Dahlan I, Massoma LD (2016) Alchornea Cordifolia, a special plant for traditional medicine: a review. J Agroecol Nat Res Manag 3(2):140-144

Ngono Ngane RA, Koanga Mogtomo ML, Tchinda Tiabou A, Magnifouet Nana H, Motso Chieffo PR, Mballa Bounou Z, Ebelle Etame RM, Ndifor F, Biyiti L (2011) Amvam Zollo PH (2011) Ethnobotanical survey of some Cameroonian plants used for treatment of viral diseases. Afr J of P1 Sci 5(1):15-21

Ngouela S, Tsamo E, Connolly JD (1994) Lignans and other constituents of Zanthoxylum heitzii. Phytochemistry 37(3):867-869

Ngoupayo J, Ebene Moukoury AM, Mushagalusa Kasali F, Kourouma K, Ntsama Essomba C (2018) Preliminary phytochemical screening and antimicrobial evaluation of leaves and barks extracts from Cola anomala (Schott and Endlicher). J Pharmacogn Phytochem 7(5):2262-2266

Njayou FN, Moundipa PF, Tchana AN, Ngadjui BT, Tchouanguep FM (2008) Inhibition Of microsomal lipid peroxidation and protein oxidation by extracts from plants used in Bamun Folk medicine (Cameroon) against hepatitis. African J Tradit Complem Alter Med 5(3):278-289

Noumedem JAK, Mihasan M, Lacmata ST, Stefan M, Kuiate JR (2013) Kuete, V (2013) Antibacterial activities of the methanol extracts of ten Cameroonian vegetabLs against Gram-negative multidrugresistant bacteria. BMC Complement Altern Med 13:26

Noumi N, Manga PN (2011) Traditional medicines for HIV/AIDS and opportunistic infections in North-West Cameroon: case of skin infections. Am J Trop Med Pub Health 1(3):44-64

Ntie-Kang F, Likowo Lifongo L, Mevaa Mbaze L, Ekwelle N, Owono Owono LC, Megnassan E, Judson PN, Sippl W, Efange SMN (2013) Cameroonian medicinal plants: a bioactivity versus 
ethnobotanical survey and chemotaxonomic classification. BMC Compl Alter Med 13:147

Oguntibeju OO (2018) (2018) Medicinal plants with anti-inflammatory activities from selected countries and regions of Africa. J Infl Res 11:307-317

Ogunwande I, Avoseh ON, Igile DO, Lawal OA, Ascrizzi R, Guido F (2019) Chemical constituents, anti-nociceptive and anti-inflammatory activities of essential oil of Phyllanthus muellerianus. Nat Prod Com 2019:1-7

Oladele JOO, Ajayi EI, Oyedotun MO, Oluwaseun TO, Boyede DO, Boluwaji MA, Olu IO (2020) Adenike TO 2020 A systematic review on COVID-19 pandemic with special emphasis oncurative potentials of Nigeria based medicinal plants. Heliyon 6:e04897

Oldham P, Colin B, Stephen H (2013). Biodiversity in the Patent System: Cameroon. A country study of genetic resources and traditional knowledge in the patent system of relevance to Cameroon. Report prepared for Deutsche Gesellschaft für Internationale Zusammenarbeit (GIZ)

Oumar M, Tume C, Kamanyi A (2010) Immunostimulation by Stem Barks of Pseudocedrela kotschyi (Family: Meliaceae): stimulation of phagocytosis activities of macrophages and proliferative response of lymphocytes by aqueous, methanol and hexane extracts. Int J Sci Eng Res 8(11)

Packman EW, Abbott DD, Harrisson JW (2006) A preliminary study of the pharmacology of 11-desmethoxyreserpine (raunormine) an alkaloid from Rauwolfia canescens. J Am Pharma Assoc 45(2 Part 1):89-93

Papu S, Jalvir S, Sweta S, Singh BR (2014) Medicinal value of garlic (Allium sativum L.) in human life: an overview. Greener J Agri Sci 2(6):265-280

Pone Kamdem B (2017) Advances on ethnomedicinal uses, phytochemistry, and pharmacology of spathodea campanulata P. Beauv. EC Pharmacol Toxicol 5(2):51-62

Poudel Adhikari S, Meng S, Wu Y, Mao Y, Ye R, Wang Q, Sun C, Sylvia S, RozelL S, Raat H, Zhou HA (2020) Literature review of 2019 novel coronavirus during the early outbreak period: epidemiology, causes, clinical manifestation and diagnosis, prevention and control. Preprints 2020:77-137

Prompetchara E, Ketloy C, Palaga T (2020) Immune responses in COVID-19 and potential vaccines: lessons learned from SARS and MERS epidemic. Asian Pac J Allergy Immunol 38:1-9

Rahmani AH, Almatroudi A, Alrumaihi F, Khan AA (2018) Pharmacological and therapeutic potential of neem (Azadirachta indica). Phcog Rev 12:250-255

Rhazri KS, Hamri M, Lbri R, Onguén H, Ouchetto A, Hafid M, Khouili M (2015) Isolation of 3,5,7,4'-Tetrahydroxyflavone (Kaempferol) from the methanol extract of the Laves of Cassia alata and invitro evaluation of its potential inhibitory effect on the human pulmonary arterial hypertension (PAH). Der Pharma Chem 7(7):241-245

Sadeer BN, Llorent-Martínez EJ, Bene K, Fawzi Mahomoodally M, Mollica A, Ibrahime Sinan K, Stefanucci A, Ruiz-Riaguas A, Fernández-de Córdova ML, Zengin G (2019) Chemical profiling, antioxidant, enzyme inhibitory and molecular modelling studies on the leaves and stem bark extracts of three African medicinal plants. J Pharm Biomed Anal 10(174):19-33

Sagnia B, Fedeli D, Casetti R, Montesano C, Falcioni G et al (2014) Antioxidant and Anti Inflammatory Activities of Extracts fromCassia alata, Eleusine indica, Eremomastax speciosa, Carica papaya and Polyscias fulva Medicinal Plants Collected in Cameroon. PLoS ONE 9(8):e103999

Sahu P, Giri D, Singh R, Pandey P, Gupta S, Shrivastava A, Pandey Kumar AK (2014) Therapeutic and medicinal uses of Aloe vera: a review. Pharmacol Pharm 4(8):599-610

Saini S, Kaur H, Verma B, Singh SK (2009) Kigelia africana (Lam.) Benth.—an overview. Nat Prod Rad. 8(2):190-197
Sakah Kaunda J, Zhang YJ (2019) The Genus Solanum: an ethnopharmacological, phytochemical and biological properties review. Nat Prod Bioprospect 9(2):77-137

Sani I, Abdulhamid A, Bello F (2014) Eucalyptus camaldulensis: Phytochemical composition of ethanolic and aqueous extracts of the leaves, stembark, root, fruits and seeds. J Sci Innov Res 3(5):523-526

Sebua SS, Maroyi M (2013) Medicinal plants used for the treatment of tuberculosis by Bapedi Traditional healers in three districts of the Limpopo province, south Africa. Afr J Tradit Complement Altern Med 10(2):316-323

Shafei NKA, Elshafie AE, Nour A (2016) Antitoxic, Antifungal and phytochemical analysis of medicinal compounds of Guiera senegalensis Leaves in Sudan. J Plant Biochem Physiol 4:166

Shaghaghi N (2020) Molecular Docking study of novel COVID19 Protease with low risk TerpenoidesCompounds of Plants. ChemRxiv. Preprint. Accessed on April 22, 2020

Shakya AK (2016) Medicinal plants: Future source of new drugs. Int J Herb Med 4(4):59-64

Sharma AD, Kaur I (2020) Jensenone from eucalyptus essential oil as a potential inhibitor of COVID 19 corona virus infection. Res Rev Biotechnol Biosci 7(1):59-66

Shio-Shin J, Ping-Ing L, Po-Ren H (2020) Treatment options for COVID-19: the reality and challenges. J Microbiol Immunol Infect 53:436-443

Sidjui Sidjui L, Melong R, Mahiou-Lddet V, Herbette G, Tiabou Tchinda A, Ollivier E, Ngosong Folefoc G (2015) Triterpenes and Lignans from Kigelia Africana. J Appl Pharma Sci 5(2):001-006

Sputniknews (2020) Au Burkina Faso, Ls phytomédicaments de l'espoir contre L COVID-19. Retrieved from https://fr.sputn iknews.com/afrique/202004031043470136-au-burkina-fasoLs-phytomedicaments-de-Lspoir-contre-L-COVID-19/, 21 april 2020.

Taghizadeh-Hesary F, Akbari H (2020) The powerful immune system against powerful COVID-19: a hypothesis. Med Hypoth

Taghreed AH, Murad AM, Mohamed AD, Saleh AQ (2017) Antiviral activities of Capsicum annuum methanolic extract against herpes simplex virus 1 and 2. Pak J Zool 49(1):267-272

Tala Sipowo RV, Wache Ouahouo BM, Djomkam Maza HL, Ishikawa H, Nishino H, Mkounga PN (2017) Triterpenes and coumaroyltyramide from Ochthocosmusafricanus. J Diseases Med Pl 3(1):12-16

Tamo SP, Essama RSH, Etoa FX (2016) Plants used in Bandjoun village (La'Djo) to cure infectious diseases: an ethnopharmacology survey and in-vitro Time-Kill Assessment of some of them against Escherichia coli. The J Phytopharmacol 5(2):56-70

Tatsimo SJN, Tamokou JdD, Havyarimana L et al (2012) Antimicrobial and antioxidant activity of kaempferol rhamnoside derivatives from Bryophyllum pinnatum. BMC Res Notes 5:158. https://doi. org/10.1186/1756-0500-5-158

Tchuenguem RT, Kechia FA, Kuiate JR, Dzoyem JP (2017) Ethnopharmacological survey, antioxidant and antifungal activity of medicinal plants traditionally used in Baham locality (Cameroon) to treat fungal infections. Arch Med Biomed Res 3(2):91-103

Tene M, Tane P, Sondengam BL (2004) Connolly JD (2004) Lignans from the roots of Echinops giganteus. Phytochemistry 5(4):2101-2105

Tijani AY, Okhale SE, Salawu TA, Onigbanjo HO, Obianodo LA, Akingbasote JA, Salawu OA, Okogun JI, Kunl FO, Emeje M (2009) Antidiarrhoeal and Antibacterial properties of crude aqueous stem bark extract and fractions of Parkia biglobosa (Jacq.) R. Br. Ex G. Don. Afr J Pharma Pharmacol 3(7):347-353

Tinitana F, Rios M, Romero-Benavides JC, de la Cruz Rot M, Pardode-Santayana M (2016) Medicinal plants sold at traditional markets in southern Ecuador. J Ethnobiol Ethnomed 12(1) 
Titanji VPK, Zofou D, Ngemeneya MN (2008) The antimalarial potential of medicinal plants used for the treatment of malaria in Cameroonian Folk Medicine. Afr J Trad CAM 5(3):302-321

Tomar A (2017) Medicinal use of Abelmoschus esculentus (Linn.) Moench. (Bhindi) to cure fever. J Phcog Phytochem 6(4):596-597

Tsabang N, Fokou PVT, Tchokouaha LRY, Noguem B, Bakarnga-Via I, Nguepi MSD, Nkongmeneck BA, Boyom FF (2012) Ethnopharmacological survey of Annonaceae medicinal plants used to treat malaria in four areas of Cameroon. $\mathrm{J}$ Ethnopharmacol 139(1):171-180

Tsobou R, Mapongmetsem P-M, Voukeng KI (2015) Van Damme P (2015) Phytochemical screening and antibacterial activity of medicinal plants used to treat typhoid fever in Bamboutos division, West Cameroon. J Appl Pharma Sci 5(06):034-049

Vellingiri B, Jayaramayya K, Iyer M, Narayanasamy A, Govindasamy V, Giridharan G, Ganesan S, Venugopal A, Venkatesan D, Ganesan H, Rajagopalan K, Pattanathu KSMR, Cho S, Kumar NS, Subramaniam MD (2020) COVID-19: a promising cure for the global panic. Sce Total Envion 725(10): 138277

Wu R, Wang L, Dina Kuo HC, Shannar A, Peter R, Chou PJ, Li S, Hudlikar R, Liu X, Liu Z, Poiani GJ, Amorosa L, Brunetti L, Kong AN (2020) An update on current therapeutic drugs treating COVID-19. Curr Pharmacol Rep 6:56-70
Yemele MD, Telefo PB, Lienou LL, Tagne SR, Fodouop CSP, Goka CS, Lmfacka MC, Moundipa FP (2014) Ethnobotanical survey of medicinal plants used for pregnant women's health conditions in Menoua division-West Cameroon. J Ethnopharmacol 160:14-39

Yinyang J, Mpondo Mpondo E, Tchatat M, Ndjib RC, Mvogo Ottou PB, Dibong SD (2014) Les plantes à alcaloïdes utilisées par les populations de la ville de Douala (Cameroun). J Appl Biosci 78:6600-6619

Zhang X (1996) Traditional medicine and WHO. World Health No. 2

Zhang D, Wu K, Zhang X, Deng S (2020) Peng B (2020) In silico screening of Chinese herbal medicines with the potential to directly inhibit 2019 novel coronavirus. J Integr Med 18:152-158

Zumla A, Hui DS, Azhar EI, Memish ZA, Maeurer M (2020) Reducing mortality from 2019-nCoV: host-directed therapies should be an option. Correspondence 395(10224):PE35-PE36

Publisher's Note Springer Nature remains neutral with regard to jurisdictional claims in published maps and institutional affiliations. 